\title{
Stability and Stabilization of Semi-Markov Jump Linear Systems with Exponentially Modulated Periodic Distributions of Sojourn Time
}

\author{
Lixian Zhang, Senior Member, IEEE, Ting Yang, Patrizio Colaneri, Fellow, IEEE
}

\begin{abstract}
This paper is concerned with a class of discretetime semi-Markov jump linear systems (S-MJLSs) subject to exponentially modulated periodic (EMP) probability density function (PDF) of sojourn time, and the problems of stability and stabilization are addressed. Setting a relatively large period, the considered systems are capable of approximating the general S-MJLSs (without any requirements on sojourn time PDFs) for which numerically testable stability and stabilization conditions are rather difficult to obtain. Necessary and sufficient criterion for mean square stability of the general S-MJLSs are first derived, which involve an infinite number of conditions and as such not checkable. However, the developments lay a foundation to further develop the numerically testable conditions for the systems when the PDF of sojourn time are EMP albeit the sojourn time can tend to infinity. The derivations explicitly depend on the PDF of sojourn time, which circumvents the difficulty in obtaining the memory transition probabilities of SMJLSs. The adopted Lyapunov function is sojourn-time-dependent (STD), by which the existence conditions of STD controller are developed as well using certain techniques that can eliminate the terms of power of matrices in the stability conditions. Two illustrative examples including a class of population ecological systems are presented to show the validity and applicability of the developed theoretical results.
\end{abstract}

Index Terms-Exponentially modulated periodic distribution; Mean squre stability; Probability density functions; Semi-Markov jump linear systems; Sojourn time.

\section{INTRODUCTION}

Stochastic switching systems are generally referred to as those dynamic systems displaying autonomous and stochastic variations among a finite number of system modes that are represented by differential/difference equations. The phenomena of stochastic switching can be typically found in various electric circuit systems [1], [2], [3], multi-legged and omnidirectional mobile robots [4], [5], and biological systems [6], [7], communication networks [8], [9], [10]. As an important class of stochastic switching systems, Markov jump linear systems (MJLSs) have been extensively investigated over the past decades, and the adopted Markov process or Markov chain can greatly facilitate the analysis and synthesis of the MJLSs. To date, many systematic results on such systems are available in the literature, see for example, [11], [12]. In particular, necessary and sufficient conditions for some control

L. Zhang and T. Yang are with the School of Astronautics, Harbin Institute of Technology, Harbin, 150080, China. (e-mail: lixianzhangehit.edu.cn; tingyangahit.edu.cn)

Patrizio Colaneri is with the Dipartimento di Elettronica e Informazione, Politec nico di Milano, Piazza Leonardo da Vinci 32, 20133 Milano, Italy. (e-mail : colaneri@elet.polimi.it). issues of the systems have been obtained, such as stability analysis for the cases when the transition probabilities (TPs) are completely known [13], [14] or not [15], and $H_{\infty}$ analysis and control problems [16], [17], etc. It should be emphasized that the developed theories can be effectively applied only if the stochastic switching can be indeed modeled by Markov process or Markov chain where the TPs are memoryless.

To generalize the scope of stochastic switching systems, a naturally extended research frontier is the semi-Markov jump systems (S-MJLSs) where the TPs will be memory, i.e., the $\mathrm{TP}$ at each time depends on all the history information of elapsed switching sequences. In mathematics, the sojourn time between the consecutive jumps in S-MJLSs is not necessarily subject to exponential or geometry distribution (continuoustime or discrete-time domain, respectively) like in the cases of MJLSs. However, the generality of semi-Markov chain in the capability of modeling stochastic switching unavoidably lead to the considerable complexity of studying the S-MJLSs, even the basic stability and stabilization issues. Though some advances have been achieved so far, see for example, [18], [19], [20] by considering special classes of probability density functions (PDFs), quite a few key problems are still largely open, such as the fundamental necessary and sufficient stability criteria and numerically checkable conditions in solving stabilizing controller, despite a recent attempt to take upper bounds on the sojourn time that actually will not tend to infinity in practice [21].

In essence, the obstacles to the above-mentioned problems lie in that the TPs of a semi-Markov chain are memory, therefore the computations of such time-varying TPs will involve infinite iterations. As a consequence, the desired necessary and sufficient conditions have not been developed paralleling the results for MJLSs. Also, without any approximation on the memory TPs or any truncation on the sojourn time to be above bounded, in general it will be rather difficult to obtain a finite of numerically testable stability conditions for S-MJLSs. However, a noteworthy exception is the case when the sojourn time is subject to exponentially modulated periodic (EMP) distribution. Notice that by setting up a relatively large period and transforming any types of non-EMP PDFs to be EMP, the original S-MJLSs can be approximated by the transformed systems for which only a limited number of conditions are required (benefited from the periodicity). It can be conjectured that, in this setting, the designed controller for the transformed S-MJLSs can be effective for the original systems since the PDFs of sojourn time are completely equivalent in the first 
period.

Motivated by the aforementioned observations, this paper focuses on the stability and stabilization problems for the discrete-time semi-Markov jump linear systems (S-MJLSs) with EMP PDF of sojourn time. The contributions of this paper lie in that: (i) Necessary and sufficient stability conditions are derived for general S-MJLSs (sojourn time is not necessarily subject to EMP distribution) in the sense of mean square stability (MSS). The obtained criterion lays a foundation to further develop the numerically testable conditions for the systems when the PDF of sojourn time are EMP (albeit the sojourn time can tend to infinity). (ii) The derivations explicitly depend on the PDF of sojourn time, which circumvents the difficulty in obtaining the memory transition probabilities of S-MJLSs. (iii) The adopted Lyapunov function is sojourn-time-dependent (STD), which is less conservative than the sojourn-time-independent ones, and as such the STD stabilizing controller can be designed as well while using certain techniques to eliminate the terms of power of matrices in the stability conditions. The remainder of this paper is organized as follows. In Section II, the problem formulation is presented and the purposes of the paper are stated. The detailed derivations of the proposed results are given in Section III. Two numerical examples are provided to verify the theoretical findings in Section IV, and Section V concludes this paper.

Notations: In this paper, $\mathbb{R}^{n}$ and $\mathbb{R}^{n \times m}$ denotes the $n$ dimensional Euclidean space and the set of all $n \times m$ real matrices, respectively; $\|\cdot\|$ refers to the Euclidean vector norm; $\mathbb{N}$ and $\mathbb{N}_{+}$denote the set of non-negative integers and the set of positive integers, respectively; $\mathbb{N}_{\geq s_{1}}, \mathbb{N}_{\leq s_{1}}$ and $\mathbb{N}_{\left[s_{1}, s_{2}\right]}$ denote the sets $\left\{k \in \mathbb{N}_{+} \mid k \geq s_{1}\right\},\left\{k \in \mathbb{N}_{+} \mid k \leq s_{1}\right\}$ and $\left\{k \in \mathbb{N}_{+} \mid s_{1} \leq k \leq s_{2}\right\}$, respectively, for some $s_{1}, s_{2} \in \mathbb{Z}_{+}$. A real-valued scalar function $\alpha: \mathbb{R}_{+} \rightarrow \mathbb{R}_{+}$that is continuous, strictly increasing, and has $\alpha(0)=0$ is said to be of class $\mathcal{K}$. For notation $(\Psi, \mathcal{F}, \operatorname{Pr}), \Psi$ represents the sample space, $\mathcal{F}$ is the $\sigma$-algebra of subsets of the sample space, and $\operatorname{Pr}$ is the probability measure on $\mathcal{F}$. $X^{\prime}$ and $\mathbb{E}[X]$ stand for the transpose and mathematical expectation of matrix $X$, respectively. The notation $P \succ 0(\succeq 0)$ means $P$ is real symmetric positive (semi-positive) definite. In addition, $\operatorname{diag}\{\cdots\}$ and $\operatorname{diag}_{(n)}\{X\}$ stand for a block-diagonal matrix and a $n \times n$ block-diagonal matrix where all diagonal entries are $X$, respectively. Symbol $*$ is used as an ellipsis for the terms that are introduced by symmetry. $\mathbf{I}$ and $\mathbf{0}$ represent the identity matrix and zero matrix, respectively. Matrices, if their dimensions are not explicitly stated, are assumed to be compatible for algebraic operations.

\section{Preliminaries and Problem Formulation}

Fix the complete probability space $(\Psi, \mathcal{F}, \operatorname{Pr})$ and consider the following discrete-time stochastic switching systems:

$$
x(k+1)=A\left(r_{k}\right) x(k)+B\left(r_{k}\right) u(k)
$$

where $x(k) \in \mathbb{R}^{n}, u(k) \in \mathbb{R}^{n_{u}}$ are the system state and control input, respectively; $\left\{r_{k}\right\}_{k \in \mathbb{N}_{+}}$is a stochastic process and considered to be a semi-Markov chain, which takes values in a finite set $\mathcal{I} \triangleq\{1,2, \ldots, M\}$, and governs the switching among $M$ system modes. For $r_{k}=i \in \mathcal{I}$, the pair of matrices of the $i^{\text {th }}$ system mode is denoted by $\left(A_{i}, B_{i}\right)$, which are real known matrices.

To recall the formal definition of semi-Markov chain, it is needed to first present the concept of Markov renewal chain that involves three stochastic processes attached to $\left\{r_{k}\right\}_{k \in \mathbb{N}_{+}}$,

(i) the stochastic process $\left\{k_{n}\right\}_{n \in \mathbb{N}_{+}} \in \mathbb{N}_{+}$, where $k_{n}$ denotes the time at the $n^{t h}$ jump of $r_{k}$. It is noted that $k_{0}=0$, and $k_{n}$ increases monotonically with $n$.

(ii) the stochastic process $\left\{R_{n}\right\}_{n \in \mathbb{N}_{+}} \in \mathcal{I}$, where $R_{n}$ is the mode index of $r_{k}$ at the $n^{\text {th }}$ jump and $R_{0} \in \mathcal{I}$ is the initial state.

(iii) the stochastic process $\left\{S_{n}\right\}_{n \in \mathbb{N}_{+}} \in \mathbb{N}_{+}$, where $S_{n}=$ $k_{n}-k_{n-1}, \forall n \in \mathbb{N}_{+}$denotes the sojourn time of mode $R_{n-1}$ between the $(n-1)^{t h}$ jump and $n^{t h}$ jump, and $S_{0}=0$.

Definition 1: [22] The stochastic process $\left\{\left(R_{n}, k_{n}\right)\right\}_{n \in \mathbb{N}_{+}}$ is said to be a discrete-time homogeneous Markov renewal chain (MRC) if the following holds $\forall i, j \in \mathcal{I}, \forall \tau \in \mathbb{N}_{+}$and $\forall n \in \mathbb{N}_{+}$:

$$
\begin{aligned}
& \operatorname{Pr}\left(R_{n+1}=j, S_{n+1}=\tau \mid R_{0}, \ldots, R_{n}=i ; k_{0}, \ldots, k_{n}\right) \\
= & \operatorname{Pr}\left(R_{n+1}=j, S_{n+1}=\tau \mid R_{n}=i\right) \\
= & \operatorname{Pr}\left(R_{1}=j, S_{1}=\tau \mid R_{0}=i\right) .
\end{aligned}
$$

In addition, $\left\{R_{n}\right\}_{n \in \mathbb{N}_{+}}$is called the embedded Markov chain (EMC) of MRC $\left\{\left(R_{n}, k_{n}\right)\right\}_{n \in \mathbb{N}_{+}}$, and the transition probabilities (TPs) of $\left\{R_{n}\right\}_{n \in \mathbb{N}_{+}}$are defined by $\theta_{i j} \triangleq$ $\operatorname{Pr}\left(R_{n+1}=j \mid R_{n}=i\right), \forall i, j \in \mathcal{I}$ with $\theta_{i i}=0$.

Definition 2: [22] Consider a MRC $\left\{\left(R_{n}, k_{n}\right)\right\}_{n \in \mathbb{N}_{+}}$. The stochastic process $\left\{r_{k}\right\}_{k \in \mathbb{N}_{+}}$is said to be a semi-Markov chain (SMC) associated with MRC $\left\{\left(R_{n}, k_{n}\right)\right\}_{n \in \mathbb{N}_{+}}$, if $r_{k}=$ $R_{N(k)}, \forall k \in \mathbb{N}_{+}$, where $N(k) \triangleq \max \left\{n \in \mathbb{N}_{+} \mid k_{n} \leq k\right\}$.

Unlike Markov jump systems in which a Markov chain governs the switching of system modes and as such the memoryless TPs are sufficient for control issues, for a semiMarkov jump system, it is necessary to introduce the following definitions and related properties for later derivations.

Definition 3: [22] Given a SMC $\left\{r_{k}\right\}_{k \in \mathbb{N}_{+}}$corresponding to a $\operatorname{MRC}\left\{\left(R_{n}, k_{n}\right)\right\}_{n \in \mathbb{N}_{+}}$,

(i) the sojourn-time probability density function (PDF) depending on both the current mode $i$ and the next system mode $j$ is defined as

$$
\begin{array}{r}
\omega_{i j}(\tau) \triangleq \operatorname{Pr}\left(S_{n+1}=\tau \mid R_{n+1}=j, R_{n}=i\right) \\
\forall j \neq i, i, j \in \mathcal{I}, \forall \tau \in \mathbb{N}_{+}
\end{array}
$$

and $\omega_{i i}(\tau)=0, \forall i \in \mathcal{I}, \forall \tau \in \mathbb{N}_{+}$.

(ii) the semi-Markov kernel $\Pi(\tau)=\left[\pi_{i j}(\tau)\right]_{i, j \in \mathcal{I}} \in \mathbb{R}^{M \times M}$ where $\pi_{i j}(\tau)$ is defined as

$$
\begin{aligned}
\pi_{i j}(\tau) & \triangleq \operatorname{Pr}\left(R_{n+1}=j, S_{n+1}=\tau \mid R_{n}=i\right) \\
& =\theta_{i j} \omega_{i j}(\tau), \forall j \neq i, i, j \in \mathcal{I}, \forall \tau \in \mathbb{N}_{+}
\end{aligned}
$$

and $\pi_{i i}(\tau)=0, \forall i \in \mathcal{I}, \forall \tau \in \mathbb{N}_{+}$.

(iii) the sojourn-time PDF only depending on the current mode $i$ is defined as

$$
\begin{aligned}
f_{i}(\tau) & \triangleq \operatorname{Pr}\left(S_{n+1}=\tau \mid R_{n}=i\right) \\
& =\sum_{j \in \mathcal{I}} \theta_{i j} \omega_{i j}(\tau), \forall i \in \mathcal{I}, \forall \tau \in \mathbb{N}_{+}
\end{aligned}
$$


(iv) the sojourn-time cumulative density function (CDF) in the current system mode $i$ is defined as

$$
\begin{aligned}
F_{i}(\tau) & \triangleq \operatorname{Pr}\left(S_{n+1} \leq \tau \mid R_{n}=i\right) \\
& =\sum_{l=0}^{\tau} \sum_{j \in \mathcal{I}} \pi_{i j}(\tau) \\
& =\sum_{l=0}^{\tau} \sum_{j \in \mathcal{I}} \theta_{i j} \omega_{i j}(l), \forall i \in \mathcal{I}, \forall \tau \in \mathbb{N}_{+} .
\end{aligned}
$$

It is assumed that $\omega_{i j}(0)=\pi_{i j}(0)=f_{i}(0)=F_{i}(0)=0$, $\forall j \neq i, i, j \in \mathcal{I}$ without loss of generality.

(v) the TPs of $\left\{R_{n}\right\}$ involving the currently-known sojourn time are defined as

$$
\begin{aligned}
\lambda_{i i}(\tau) & \triangleq \operatorname{Pr}\left(S_{n+1}>\tau \mid R_{n}=i, S_{n+1}>\tau-1\right) \\
& =\frac{1-F_{i}(\tau)}{1-F_{i}(\tau-1)}, \\
\lambda_{i j}(\tau) & \triangleq \operatorname{Pr}\left(R_{n+1}=j, S_{n+1}=\tau \mid R_{n}=i, S_{n+1}>\tau-1\right) \\
& =\frac{\pi_{i j}(\tau)}{1-F_{i}(\tau-1)}=\frac{\theta_{i j} \omega_{i j}(\tau)}{1-F_{i}(\tau-1)}, j \neq i, i, j \in \mathcal{I} .
\end{aligned}
$$

(vi) the TPs of $\left\{r_{k}\right\}$ are defined as

$$
\epsilon_{i j}(\vec{k}) \triangleq \operatorname{Pr}\left(r_{k+1}=j \mid r_{k}=i\right), i, j \in \mathcal{I}
$$

where $\vec{k} \triangleq(1,2, \cdots, k)$ indicates that the TPs of $\left\{r_{k}\right\}$ are dependent on the elapsed mode evolution (i.e., the past switching sequence). Note that we use $\epsilon_{i}(0), \forall i \in \mathcal{I}$, to denote the initial mode probability.

The computation of the TPs defined in Definition 3(vi) is given in Appendix A.

Remark 1: From Definition 3(vi), the counterpart of TPs $\epsilon_{i j}(\vec{k})$ in Markov chain are memoryless, i.e., independent of the past switching sequence, which are commonly used to obtain the criteria for control issues in MJLSs. However, in a SMC, $\epsilon_{i j}(\vec{k})$ cannot be obtained a priori, such that it is rather difficult to similarly use $\epsilon_{i j}(\vec{k})$ for the developments of the criteria. In this paper, we shall resort to the SMK $\pi_{i j}(\tau)$ in Definition 3(ii), where two stochastic variables are needed, i.e., the sojourn time of current mode and the index of next mode, for a known index of the current mode.

Remark 2: Further to Remark 1, while using $\pi_{i j}(\tau)$, the PDF $\omega_{i j}(\tau)$ of sojourn time will be directly used including the types of the distribution and the parameters. Therefore, it will outperform $f_{i}(\tau)$ that was used in quite a few existing literature, since it is straightforward that different $\omega_{i j}(\tau)$ may lead to a same $f_{i}(\tau)$ according to Definition 3(iii).

Remark 3: A natural question is about the link and difference between the Markov chain and SMC. Although details on this issue can be seen in pure mathematics literature, e.g., [23], [24], [25], here we would like to present how the two TPs $\lambda_{i j}(\tau)$ and $\epsilon_{i j}(\vec{k})$ can be reduced to constant/memoryless ones when the PDF of sojourn time is subject to the geometric distribution, i.e., for the case when $w_{i j}(\tau) \equiv w_{i}(\tau)=$ $\left(1-p_{i}\right)^{\tau-1} p_{i}, p_{i} \in(0,1), i, j \in \mathcal{I}$. First, from (2) and (3), it holds that

$$
\begin{aligned}
& \lambda_{i j}(\tau)=\frac{\theta_{i j} \omega_{i j}(l)}{1-\sum_{l=0}^{\tau-1} \sum_{j \in \mathcal{I}} \theta_{i j} \omega_{i j}(l)}=p_{i} \theta_{i j} \\
& \lambda_{i i}(\tau)=\frac{1-\sum_{l=0}^{\tau} \sum_{j \in \mathcal{I}} \theta_{i j} \omega_{i j}(l)}{1-\sum_{l=0}^{\tau-1} \sum_{j \in \mathcal{I}} \theta_{i j} \omega_{i j}(l)}=1-p_{i}
\end{aligned}
$$

Second, as for $\epsilon_{i j}(\vec{k})$, it can be also verified that

$$
\begin{aligned}
\epsilon_{i j}(\overrightarrow{1}) & =\bar{\epsilon}_{i}(0) \pi_{i j}(1) / \bar{\epsilon}_{i}(0) \\
& =\pi_{i j}(1)=\theta_{i j} w_{i j}(1)=p_{i} \theta_{i j}, j \neq i \\
\epsilon_{i i}(\overrightarrow{1}) & =1-\sum_{j \neq i, j \in \mathcal{I}} \epsilon_{i j}(\overrightarrow{1}) \\
& =1-\sum_{j \neq i, j \in \mathcal{I}} p_{i} \theta_{i j}=1-p_{i} \\
\epsilon_{j p}(\overrightarrow{2}) & =\frac{\sum_{i \neq j, i \in \mathcal{I}} \bar{\epsilon}_{i}(0) \epsilon_{i j}(1) \pi_{j p}(1)+\bar{\epsilon}_{j}(0) \pi_{j p}(2)}{\sum_{i \in \mathcal{I}} \bar{\epsilon}_{i}(0) \epsilon_{i j}(1)} \\
& =\frac{\sum_{i \neq j, i \in \mathcal{I}} \bar{\epsilon}_{i}(0) \theta_{i j} p_{i} \theta_{j p} p_{j}+\bar{\epsilon}_{j}(0) \theta_{j p}\left(1-p_{j}\right) p_{j}}{\sum_{i \neq j, i \in \mathcal{I}} \bar{\epsilon}_{i}(0) \theta_{i j} p_{i}+\bar{\epsilon}_{j}(0)\left(1-p_{j}\right)} \\
& =p_{j} \theta_{j p} \\
\epsilon_{j j}(\overrightarrow{2}) & =1-\sum_{p \neq j, p \in \mathcal{I}} \epsilon_{j p}(1) \\
& =1-\sum_{p \neq j, p \in \mathcal{I}} p_{j} \theta_{j p}=1-p_{j}
\end{aligned}
$$

The cases for $k \geq 3$ can be verified iteratively and omitted here.

To present the purposes of this paper precisely, the following stability definition is required.

Definition 4: Consider a discrete-time stochastic switching system $x_{k+1}=f\left(x_{k}, r_{k}\right)$, where $r_{k}$ is a certain stochastic process governing the system switching and taking values in $\mathcal{I}$. The system is said to be mean square stable $\left(\mathrm{MSS}^{1}\right)$ if, for any initial condition $x_{0} \in \mathbb{R}^{n}, r_{0} \in \mathcal{I}$, the following holds:

$$
\left.\lim _{k \rightarrow \infty} \mathbb{E}\left[\|x(k)\|^{2}\right]\right|_{x_{0}, r_{0}}=0
$$

From Definition 4, it can be observed that the random sojourn time can be of any length (even infinity), which will lead to untestable criteria in general if without any approximation on the memory TPs [26] or any truncation on the sojourn time to be above bounded [21]. However, if the PDF is periodic, it will be likely to obtain a finite number of conditions that would be checkable as shown in the sequel. Due to the property that the $\mathrm{CDF}$ of any distribution of sojourn time will be one, i.e., $\lim _{\tau \rightarrow \infty} F_{i}(\tau)=1$, the following definition on exponentially modulated periodic (EMP) distribution will be considered in this paper.

Definition 5: The PDF is said to be exponentially modulated periodic (EMP) if the following property holds,

$$
\omega_{i j}(t+K T)=\bar{\omega}_{i j}(t+K T) \mu_{i j}^{K T}, t \in[1, T], K \in \mathbb{N}
$$

where $\mu_{i j}$ is a known constant and $\bar{\omega}_{i j}(\cdot)$ is a $T$-period function with $\omega_{i j}(0)=\bar{\omega}_{i j}(0)=0$.

An illustration on the EMP PDF can be seen in Fig. 1, where four typical distributions are presented in Fig. 1(a), and the corresponding EMP distributions with $T=10$ are given in Fig. 1(b).

Remark 4: By Definition 5, it can be seen that when $K=0$, $\omega_{i j}(t)=\bar{\omega}_{i j}(t), t \in[1, T]$, namely, within the first period, the EMP PDF will be exactly the non-EMP PDFs as shown in Fig. 1. Therefore, by setting up a relatively large period and transforming the original non-EMP PDFs to be EMP, the original S-MJLSs can be approximated by the transformed

\footnotetext{
${ }^{1}$ In this paper, we will slightly abuse MSS as the abbreviation of either mean square stability or mean square stable
} 


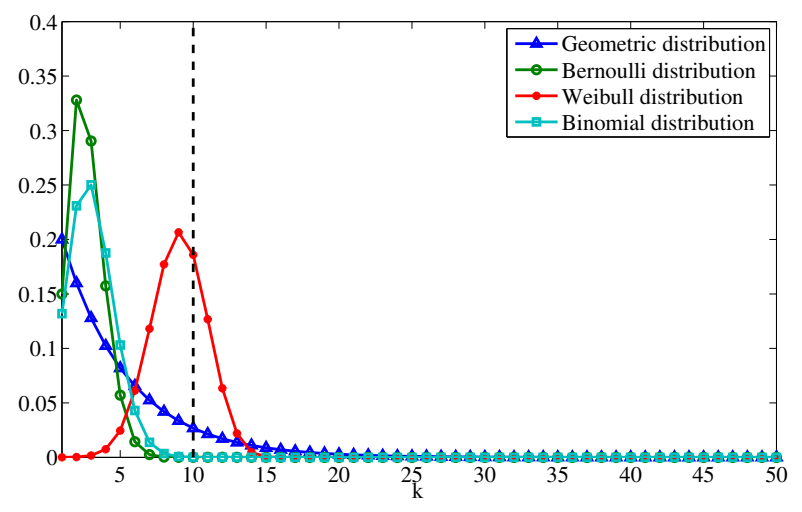

(a) Four typical distributions.

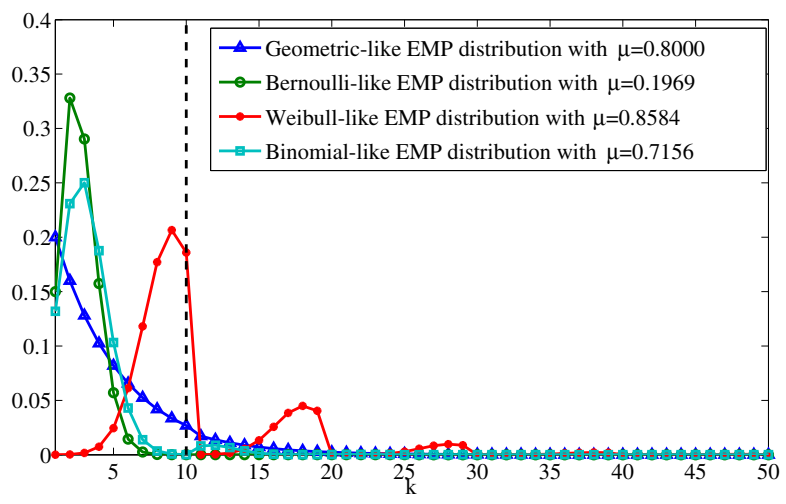

(b) The corresponding EMP distributions with $T=10$.

Fig. 1: A comparison between non-EMP and EMP distributions.

systems for which only a limited number of conditions will be required as presented in later developments. Note that in the first period, the periodic function $\bar{\omega}_{i j}(t), t \in[1, T]$ can be a general PDF without any specific type as long as $\lim _{\tau \rightarrow \infty}$ $F_{i}(\tau)=1$.

The objectives in this paper will be to derive the testable MSS criterion for system (1) with EMP PDF of sojourn time $\omega_{i j}\left(S_{n}\right)$, and to design a mode-dependent and sojourn-timedependent (STD) stabilizing controller guaranteeing the MSS of the resulting closed-loop system. The controller here is considered to be of the following form:

$$
u(k)=K_{i}\left(\alpha_{n}\right) x(k), \forall r_{k}=i \in \mathcal{I}, k \in\left[k_{n}, k_{n+1}\right)
$$

where $K_{i}\left(\alpha_{n}\right)$ is the controller gain to be determined and $\alpha_{n}$ satisfies that $S_{n}=\alpha_{n}+K T, K \in \mathbb{N}$.

For this purpose, the stability and stabilization problems for system (1) with general types of sojourn time $\omega_{i j}\left(S_{n}\right)$ will be investigated first and the corresponding mode-dependent STD stabilizing controller will have the form:

$$
u(k)=K_{i}\left(S_{n}\right) x(k), \forall r_{k}=i \in \mathcal{I}, k \in\left[k_{n}, k_{n+1}\right)
$$

where $K_{i}\left(S_{n}\right)$ is the controller gain to be determined.

\section{MAIN RESULTS}

In this section, we first consider the S-MJLSs without limitations on the PDF of sojourn time and derive the corresponding necessary and sufficient stability and stabilization criteria. Then, a set of sufficient conditions will be given, which will be applied to a special class of S-MJLSs with EMP PDF of sojourn time such that the derived theorems are testable. The relationships among these criteria are shown in Fig. 7 in Appendix B.

\section{A. Criteria for S-MJLSs with general PDF of sojourn time}

The following proposition presents the necessary and sufficient conditions ensuring that the S-MJLS (1) with general PDF of sojourn time is MSS.

Proposition 1: Consider S-MJLS (1) with $u(k) \equiv 0$. The system with general PDF of sojourn time is MSS if and only if there exist a constant $\epsilon>0$, and a set of symmetric matrices
$\left\{P_{i}(n, v) \succ 0\right\}, i \in \mathcal{I}, n \in \mathbb{N}, v \in \mathbb{N}$ such that the following inequality holds for all $i \in \mathcal{I}, n \in \mathbb{N}_{+}, \alpha \in \mathbb{N}_{[1, \infty)}, \beta \in \mathbb{N}_{+}$

$$
\sum_{\beta \in \mathbb{N}_{+}} \sum_{j \in \mathcal{I}} \tilde{\eta}_{i j}(\beta)\left(A_{i}^{\prime}\right)^{\beta} P_{j}(n+1, \beta) A_{i}^{\beta}-P_{i}(n, \alpha) \prec-\epsilon \mathbf{I}(7)
$$

where $\tilde{\eta}_{i j}(\beta) \triangleq \pi_{i j}(\beta), j \neq i, \widetilde{\eta}_{i i}(\infty) \triangleq 1-$ $\sum_{\beta \in \mathbb{N}_{+}} \sum_{j \neq i \in \mathcal{I}} \widetilde{\eta}_{i j}(\beta)$, and $\widetilde{\eta}_{i i}(\beta) \triangleq 0, \beta \in \mathbb{N}_{[1, \infty)}$.

Proof. (i) Sufficiency.

Consider the following stochastic Lyapunov function candidate for system (1).

$$
\left.V\left(n, x_{k}, r_{k_{n}}, S_{n}\right)\right|_{r_{k_{n}}=i}=x_{k}^{\prime} P_{i}\left(n, S_{n}\right) x_{k}, k \in\left(k_{n-1}, k_{n}\right] .
$$

An illustration of the above Lyapunov function candidate that is sojourn-time-dependent (STD) is given in Fig. 2.

According to (7), the following equation holds for all $r_{k_{n}}=$ $i \in \mathcal{I}, n \in \mathbb{N}_{+}, S_{n} \in \mathbb{N}_{[1, \infty)}$

$$
\begin{aligned}
& \left.\mathbb{E}\left[V\left(n+1, x_{k_{n+1}}, r_{k_{n+1}}, S_{n+1}\right)\right]\right|_{x_{k_{n}}, r_{k_{n}}, S_{n}} \\
& -V\left(n, x_{k_{n}}, r_{k_{n}}, S_{n}\right) \\
= & x_{k_{n}}^{\prime}\left(\sum_{S_{n+1} \in \mathbb{N}_{+}} \sum_{j \in \mathcal{I}} \widetilde{\eta}_{i j}\left(S_{n+1}\right)\left(A_{i}^{\prime}\right)^{S_{n+1}}\right. \\
& \left.\times P_{j}\left(n+1, S_{n+1}\right) A_{i}^{S_{n+1}}-P_{i}\left(n, S_{n}\right)\right) x_{k_{n}} \\
< & -\epsilon x_{k_{n}}^{\prime} x_{k_{n}} .
\end{aligned}
$$

Note that the above inequality also holds for the case $n=$ $0\left(S_{0}=0\right)$ since it is trivial to find $P_{i}(0,0)$ such that $(7)$ is satisfied. Therefore, one has

$$
\begin{aligned}
x_{k_{n}}^{\prime} x_{k_{n}}< & -\frac{1}{\epsilon}\left\{\left.\mathbb{E}\left[V\left(n+1, x_{k_{n+1}}, r_{k_{n+1}}, S_{n+1}\right)\right]\right|_{x_{k_{n}}, r_{k_{n}}, S_{n}}\right. \\
& \left.-V\left(n, x_{k_{n}}, r_{k_{n}}, S_{n}\right)\right\} .
\end{aligned}
$$

Taking the sum from $n=0$ to $N$ for both sides of the above inequality, we have

$$
\begin{aligned}
& \left.\mathbb{E}\left[\sum_{n=0}^{N} x_{k_{n}}^{\prime} x_{k_{n}}\right]\right|_{x_{k_{0}}, r_{k_{0}}, S_{0}} \\
< & -\frac{1}{\epsilon}\left\{\left.\mathbb{E}\left[V\left(N, x_{k_{N}}, r_{k_{N}}, S_{N}\right)\right]\right|_{x_{k_{0}}, r_{k_{0}}, S_{0}}\right. \\
& \left.-V\left(0, x_{k_{0}}, r_{k_{0}}, S_{0}\right)\right\} .
\end{aligned}
$$




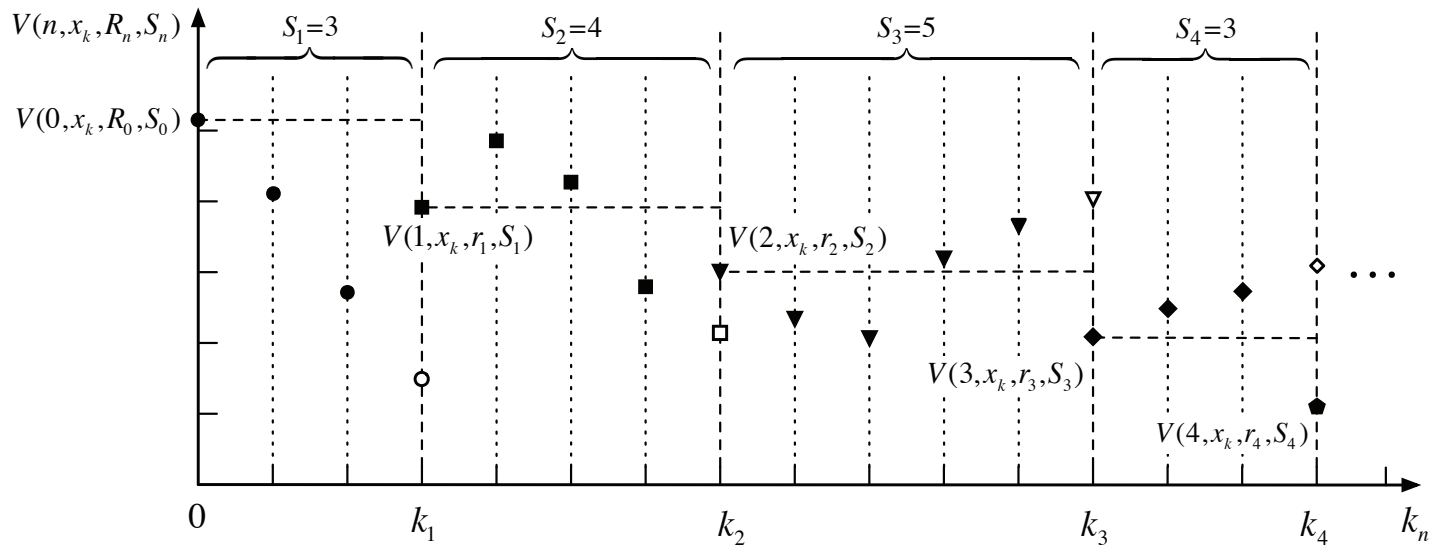

Fig. 2: An illustration of STD stochastic Lyapunov function.

When $N$ tends to $\infty$, it is straightforward from (8) that $\left.\mathbb{E}\left[\sum_{n=0}^{N} x_{k_{n}}^{\prime} x_{k_{n}}\right]\right|_{x_{k_{0}}, r_{k_{0}}}$ is finite, i.e., $\left.\lim _{n \rightarrow \infty} \mathbb{E}\left[x_{k_{n}}^{\prime} x_{k_{n}}\right]\right|_{x_{k_{0}}, r_{k_{0}}}=0$. Due to the linearity of the system, we could find a pair of class $\mathcal{K}$ functions $\alpha_{1}$ and $\alpha_{2}$, and a finite $h>0$ such that

$$
\begin{aligned}
\alpha_{1}\left(\left\|x_{k}\right\|\right) & \leq \alpha_{1}^{-1}\left(V\left(n, x_{k}, r_{k_{n}}, S_{n}\right)\right) \\
& \leq \alpha_{1}^{-1}\left(h V\left(n, x_{k_{n}}, r_{k_{n}}, S_{n}\right)\right) \\
& \leq \alpha_{2}\left(\alpha_{1}^{-1}\left(h\left\|x_{k_{n}}\right\|\right)\right.
\end{aligned}
$$

Then, it can be inferred that $\left.\lim _{k \rightarrow \infty} \mathbb{E}\left[x_{k}^{\prime} x_{k}\right]\right|_{x_{k_{0}}, r_{k_{0}}}=$ $\left.\lim _{n \rightarrow \infty} \mathbb{E}\left[x_{k_{n}}^{\prime} x_{k_{n}}\right]\right|_{x_{k_{0}}, r_{k_{0}}}=0$. The sufficiency is proved.

(ii) Necessity.

Assuming system (1) is MSS, then at the jumping instant $k_{n}$, for given positive definite matrices $Q_{r_{k_{t}}}\left(t, S_{t}\right), t \in \mathbb{N}$, one can define a sequence $\left\{x_{k_{n}}^{\prime} \widetilde{P}_{r_{k_{n}}}\left(n, S_{n}, N\right) x_{k_{n}}\right\}_{n \in \mathbb{N}_{[0, N]}}$ to be

$$
\begin{aligned}
& x_{k_{n}}^{\prime} \widetilde{P}_{r_{k_{n}}}\left(n, S_{n}, N\right) x_{k_{n}} \\
\triangleq & \left.\mathbb{E}\left[\sum_{t=n}^{N} x_{k_{t}}^{\prime} Q_{r_{k_{t}}}\left(t, S_{t}\right) x_{k_{t}}\right]\right|_{x_{k_{n}, r_{k_{n}}, S_{n}}}
\end{aligned}
$$

For $x_{k_{t}} \neq 0, t \in[n, N]$, as $N$ increases, $x_{k_{n}}^{\prime} \widetilde{P}_{r_{k_{n}}}\left(n, S_{n}, N\right) x_{k_{n}}>0$ is monotonically increasing but above bounded by the definition of MSS. Thus the following limit exists

$$
\begin{aligned}
& \lim _{N \rightarrow \infty} x_{k_{n}}^{\prime} \widetilde{P}_{r_{k_{n}}}\left(n, S_{n}, N\right) x_{k_{n}} \\
= & \left.\lim _{N \rightarrow \infty} \mathbb{E}\left[\sum_{t=n}^{N} x_{k_{t}}^{\prime} Q_{r_{k_{t}}}\left(t, S_{t}\right) x_{k_{t}}\right]\right|_{x_{k_{n}}, r_{k_{n}}, S_{n}}
\end{aligned}
$$

Since the above equation is valid for any $x_{k_{n}} \neq 0$, we denote

$$
P_{r_{k_{n}}}\left(n, S_{n}\right) \triangleq \lim _{N \rightarrow \infty} \widetilde{P}_{r_{k_{n}}}\left(n, S_{n}, N\right) \text {. }
$$

Also, from (9), it follows that $P_{r_{k_{n}}}\left(n, S_{n}\right)$ is positive definite. Thus we have

$$
\begin{aligned}
& \mathbb{E}\left[x_{k_{n}}^{\prime} P_{i}\left(n, S_{n}\right) x_{k_{n}}\right. \\
& \left.-x_{k_{n+1}}^{\prime} P_{r_{k_{n+1}}}\left(n+1, S_{n+1}\right) x_{k_{n+1}}\right]\left.\right|_{x_{k_{n}}, r_{k_{n}}=i, S_{n}} \\
= & \left.\mathbb{E}\left[x_{k_{n}}^{\prime} Q_{i}\left(n, S_{n}\right) x_{k_{n}}\right]\right|_{x_{k_{n}}, r_{k_{n}}=i, S_{n}}
\end{aligned}
$$

which implies that

$$
\begin{aligned}
& x_{k_{n}}^{\prime} P_{i}\left(n, S_{n}\right) x_{k_{n}} \\
& -\left.\mathbb{E}\left[x_{k_{n+1}}^{\prime} P_{r_{k_{n}+1}}\left(n+1, S_{n+1}\right) x_{k_{n+1}}\right]\right|_{x_{k_{n}}, r_{k_{n}}=i, S_{n}} \\
= & x_{k_{n}}^{\prime} Q_{i}\left(n, S_{n}\right) x_{k_{n}}
\end{aligned}
$$

and then

$$
\begin{aligned}
& x_{k_{n}}^{\prime} P_{i}\left(n, S_{n}\right) x_{k_{n}}-x_{k_{n}}^{\prime}\left(\sum_{S_{n+1} \in \mathbb{N}_{+}} \sum_{j \in \mathcal{I}} \widetilde{\eta}_{i j}\left(S_{n+1}\right)\right. \\
& \left.\times\left(A_{i}^{\prime}\right)^{S_{n+1}} P_{j}\left(n+1, S_{n+1}\right) A_{i}^{S_{n+1}}\right) x_{k_{n}} \\
= & x_{k_{n}}^{\prime} Q_{i}\left(n, S_{n}\right) x_{k_{n}} .
\end{aligned}
$$

Since the above is valid for any $x_{k_{n}} \neq 0, \forall n \in \mathbb{Z}$, we have

$$
\begin{aligned}
\sum_{S_{n+1} \in \mathbb{N}_{+}} & \sum_{j \in \mathcal{I}}\left[\widetilde{\eta}_{i j}\left(S_{n+1}\right)\left(A_{i}^{\prime}\right)^{S_{n+1}} P_{j}\left(n+1, S_{n+1}\right)\right. \\
& \left.\times A_{i}^{S_{n+1}}\right]-P_{i}\left(n, S_{n}\right)=-Q_{i}\left(n, S_{n}\right) \prec-\epsilon \mathbf{I}
\end{aligned}
$$

where $\epsilon \leq \inf _{i \in \mathcal{I}, n, S_{n} \in \mathbb{N}_{+}}\left\{\iota_{\min }\left(Q_{i}\left(n, S_{n}\right)\right)\right\}$ with $\iota_{\min }(M)$ denoting the minimum eigenvalue of matrix $M$. Thus the necessity is proved.

In mathematics, the probability $\widetilde{\eta}_{i i}(\infty)$ for a switching system cannot be zero, which means that the system can stay within a subsystem for an infinite time. However, a practical switching system within a control operation interval cannot cover the case that the sojourn time is infinite. Therefore, in what follows, it is assumed that $\widetilde{\eta}_{i i}(\infty)=0$ (which is consistent to $\left.\sum_{\alpha \in \mathbb{N}_{+}} \sum_{j \neq i \in \mathcal{I}} \widetilde{\eta}_{i j}(\alpha)=1\right)$. Nevertheless, only for the case that the sojourn time is subject to a deterministic upper bound, the conditions are countable and as such Proposition 1 can be tested.

It should be noted that $A_{i}^{\beta}$ contained in Proposition 1 , is not linear in $A_{i}$ and then is not suitable for controller design. To overcome the difficulty caused by the power of matrices, certain techniques will be needed and the corresponding result is given in the following corollary.

Corollary 1: Consider S-MJLS (1) with $u(k) \equiv 0$. If and only if there exist a set of symmetric matrices $O_{i}(n, \alpha, \alpha) \succ$ 0 and a set of matrices $\mathcal{O}_{i}(n, \alpha, m), i \in \mathcal{I}, n \in \mathbb{N}_{+}, \alpha \in$ $\mathbb{N}_{[1, \infty)}, m<\alpha, m \in \mathbb{N}_{[0, \infty)}$ such that the following inequality 
holds for all $i \in \mathcal{I}, n \in \mathbb{N}_{+}, \alpha, \beta \in \mathbb{N}_{[1, \infty)}, m \in \mathbb{N}_{[0, \infty)}$

$$
\begin{array}{r}
\sum_{\beta=m+1}^{\infty}\left\{A_{i}^{\prime} \mathcal{O}_{i}(n+1, \beta, m+1) A_{i}-\mathcal{O}_{i}(n+1, \beta, m)\right\} \prec 0(11) \\
\sum_{\beta=1}^{\infty} \mathcal{O}_{i}(n+1, \beta, 0)-O_{i}(n, \alpha, \alpha) \prec 0(12)
\end{array}
$$

where $\mathcal{O}_{i}(n+1, \beta, \beta) \triangleq \sum_{j \in \mathcal{I}} \pi_{i j}(\beta) O_{j}(n, \beta, \beta)$, then system (1) is MSS.

Proof. See Appendix C.

Then, the desired STD controller of the form (6) can be designed based on Corollary 1.

Theorem 1: Consider S-MJLS (1) with the controller in the form of (6). If there exist a set of symmetric matrices $H_{i}(n, \alpha, \alpha) \succ 0, \overline{\mathcal{H}}_{i}(n, m) \succ 0$, and a set of matrices $Z_{i}, U_{i}(\alpha), i \in \mathcal{I}, n \in \mathbb{N}_{+}, \alpha \in \mathbb{N}_{[1, \infty)}, m<\alpha, m \in \mathbb{N}_{[0, \infty)}$ such that the following inequalities hold for all $i \in \mathcal{I}, n \in$ $\mathbb{N}_{+}, \alpha \in \mathbb{N}_{[1, \infty)}, m<\alpha, m \in \mathbb{N}_{[0, \infty)}$

$$
\left[\begin{array}{cc}
\Phi_{i}(n+1, m+1, m+1) & 0 \\
* & \bar{\Phi}_{i}(n+1, m+1) \\
* & * \\
& \check{\mathcal{A}}_{i}(\alpha) \mathcal{L}_{i}(m+1) \\
A_{i} Z_{i}+B_{i} U_{i}(m+1) \\
-\overline{\mathcal{H}}_{i}(n+1, m)
\end{array}\right] \prec 0
$$

with

$$
\begin{gathered}
\Phi_{i}(n+1, m+1, m+1) \triangleq \mathcal{H}_{i}(n+1, m+1, m+1)-\mathcal{Z}_{i}-\mathcal{Z}_{i}^{\prime} \\
\bar{\Phi}_{i}(n+1, m+1) \triangleq \overline{\mathcal{H}}_{i}(n+1, m+1)-Z_{i}-Z_{i}^{\prime}
\end{gathered}
$$

where $\mathcal{H}_{i}(n, m, m) \triangleq \operatorname{diag}\left\{H_{1}(n, m, m), \ldots, H_{i-1}(n, m, m)\right.$, $\left.H_{i+1}(n, m, m), \ldots, H_{M}(n, m, m)\right\}, \mathcal{Z}_{i} \triangleq \operatorname{diag}\left\{Z_{1}, \ldots\right.$, $\left.Z_{i-1}, Z_{i+1}, \ldots, Z_{M}\right\}, \quad \check{\mathcal{A}}_{i}(\alpha) \triangleq \operatorname{diag}_{(M-1)}\left\{A_{i} Z_{i}+\right.$ $\left.B_{i} U_{i}(\alpha)\right\}$, and $\mathcal{L}_{i}(m) \triangleq\left[\sqrt{\pi_{i 1}(m)} \mathbf{I}, \ldots, \sqrt{\pi_{i(i-1)}(m)} \mathbf{I}\right.$, $\left.\sqrt{\pi_{i(i+1)}(m)} \mathbf{I}, \ldots, \sqrt{\pi_{i M}(m)} \mathbf{I}\right]$, then the system is MSS. Moreover, a feasible control gain is given as

$$
K_{i}(\alpha)=U_{i}(\alpha) Z_{i}^{-1}
$$

Proof. See Appendix D.

So far, the stability and stabilization criteria are obtained for S-MJLSs with general PDFs of sojourn time. Without a truncation on the sojourn time to be above bounded, the conditions in (7) cannot be tested. However, if the PDFs of sojourn time are EMP, the resulting criterion that will contain only a finite number of conditions can be obtained. Towards this purpose, the following sufficient criteria for the underlying system with general PDFs are needed.

Theorem 2: Consider S-MJLS (1) with $u(k) \equiv 0$. Then the following two statements are equivalent and the system is MSS if one of them holds.

(i) There exists a set of symmetric matrices $\left\{P_{i}(n, \alpha) \succ 0\right\}$, $i \in \mathcal{I}, n \in \mathbb{N}_{+}, \alpha \in \mathbb{N}_{[1, \infty)}$ such that the following inequality holds for all $i \in \mathcal{I}, n \in \mathbb{N}_{+}, \alpha, \beta \in \mathbb{N}_{[1, \infty)}$

$$
\sum_{j \neq i, j \in \mathcal{I}} \eta_{i j}(\beta)\left(A_{i}^{\prime}\right)^{\beta} P_{j}(n+1, \beta) A_{i}^{\beta}-P_{i}(n, \alpha) \prec 0
$$

where $\eta_{i j}(\beta) \triangleq \pi_{i j}(\beta) / \rho_{i}(\beta)$ with $\rho_{i}(\beta) \triangleq \sum_{j \neq i, j \in \mathcal{I}} \pi_{i j}(\beta)$.

(ii) There exists a set of symmetric matrices $O_{i}(n, \alpha, m) \succ$ $0, i \in \mathcal{I}, n \in \mathbb{N}_{+}, \alpha \in \mathbb{N}_{[1, \infty)}, m \leq \alpha, m \in \mathbb{N}_{[0, \infty)}$ such that the following inequalities hold for all $i \in \mathcal{I}, n \in \mathbb{N}_{+}, \alpha, \beta \in$ $\mathbb{N}_{[1, \infty)}$, and $m<\beta, m \in \mathbb{N}_{[0, \infty)}$

$$
\begin{aligned}
& \sum_{j \neq i, j \in \mathcal{I}} \eta_{i j}(\beta)\left\{A_{i}^{\prime} O_{j}(n+1, \beta, m+1) A_{i}\right. \\
&\left.-O_{j}(n+1, \beta, m)\right\} \prec 0 \\
& \sum_{j \neq i, j \in \mathcal{I}} \eta_{i j}(\beta) O_{j}(n+1, \beta, 0)-O_{i}(n, \alpha, \alpha) \prec 0
\end{aligned}
$$

where $\eta_{i j}(\beta)$ is given in (15).

Proof. See Appendix E.

Based on Theorem 2(ii), the existence conditions of stabilizing controller (6) for S-MJLS (1) are presented in the following theorem.

Theorem 3: Consider S-MJLS (1) with $u(k) \equiv 0$. If there exist a set of matrices $Z, U_{i}(\alpha)$, and a set of symmetric matrices $H_{i}(n, \alpha, m) \succ 0, i \in \mathcal{I}, n \in \mathbb{N}_{+}, \alpha \in \mathbb{N}_{[1, \infty)}, m \leq \alpha$, $m \in \mathbb{N}_{[0, \infty)}$ such that the following inequalities hold for all $i \in \mathcal{I}, n \in \mathbb{N}_{+}, \alpha, \beta \in \mathbb{N}_{[1, \infty)}$, and $m<\beta, m \in \mathbb{N}_{[0, \infty)}$.

$$
\left[\begin{array}{cc}
\widetilde{\Phi}_{i}(n+1, \beta, m+1) & \widehat{\mathcal{A}}_{i}(\alpha) \widetilde{\mathcal{L}}_{i}(\beta) \\
* & -\widetilde{\mathcal{L}}_{i}^{\prime}(\beta) \widetilde{\mathcal{H}}_{i}(n+1, \beta, m) \widetilde{\mathcal{L}}_{i}(\beta)
\end{array}\right] \prec 0(18)
$$

with

$$
\widetilde{\Phi}_{i}(n+1, \beta, m+1) \triangleq \widetilde{\mathcal{H}}_{i}(n+1, \beta, m+1)-\widetilde{\mathcal{Z}}-\widetilde{\mathcal{Z}}^{\prime}
$$

where $\widetilde{\mathcal{H}}_{i}(n+1, \beta, m) \triangleq \operatorname{diag}\left\{H_{1}(n+1, \beta, m), \ldots, H_{i-1}(n+\right.$ $\left.1, \beta, m), H_{i+1}(n+1, \beta, m), \ldots, H_{M}(n+1, \beta, m)\right\}, \widetilde{\mathcal{Z}} \triangleq$ $\operatorname{diag}_{(M-1)}\{Z\}, \widehat{\mathcal{A}}_{i}(\alpha) \triangleq \operatorname{diag}_{(M-1)}\left\{A_{i} Z+B_{i} U_{i}(\alpha)\right\}$ and $\widetilde{\mathcal{L}}_{i}(\beta) \triangleq\left[\sqrt{\eta_{i 1}(\beta)} \mathbf{I}, \ldots, \sqrt{\eta_{i(i-1)}(\beta)} \mathbf{I}, \sqrt{\eta_{i(i+1)}(\beta)} \mathbf{I}\right.$, $\left.\ldots, \sqrt{\eta_{i M}(\beta)} \mathbf{I}\right]^{\prime}$, then the system is MSS. Moreover, the admissible controller gain for (6) is given by

$$
K_{i}(\alpha)=U_{i}(\alpha) Z^{-1}
$$

\section{Proof. See Appendix F.}

Remark 5: Comparing (11), (15), and (16), one can find that the second term of either (11) or (15) $\left(\sum_{\beta=m+1}^{\infty} \mathcal{O}_{i}(n+\right.$ $1, \beta, m)$ or $\left.P_{i}(n, \alpha)\right)$ is only dependent on mode $i$, but the second term of (16) $\left(\sum_{j \neq i, j \in \mathcal{I}} \eta_{i j}(\beta) O_{j}(n+1, \beta, m)\right)$ depends on all the modes except mode $i$. Therefore, if we use $Z_{i}$ instead of $Z$ in Theorem 3 to design a controller based on (16), it will introduce terms like $Z_{i} \mathcal{O}(n, j, m) Z_{i}, i \neq j$ (not like (11) and (15), only $Z_{i} \mathcal{O}(n, i, m) Z_{i}$ will be introduced), which will lead to matrix equality constraints that unavoidably bring difficulties to the solvability of the resulting conditions. Efficient ways capable of handling such equality constraints (not the focus of this paper) can be found in literature on the related topic. 


\section{B. EMP PDF of sojourn time}

This subsection will be devoted to the developments of stability and stabilization criteria for the cases when the PDF of sojourn time is considered to be EMP.

Theorem 4: Consider the unforced S-MJLS (1) with $u(k) \equiv$ 0 and the sojourn time subject to a set of EMP distributions with the same period $T$ for each system mode. Then, the $\mathrm{S}$ MJLS (1) is MSS, if there exists a set of symmetric matrices $\mathcal{P}_{i}(\tau) \succ 0, i \in \mathcal{I}, \tau \in \mathbb{N}_{[1, T]}$ such that the following inequality holds for all $i, j \in \mathcal{I}, \vartheta, \sigma \in \mathbb{N}_{[1, T]}$

$$
\sum_{j \neq i, j \in \mathcal{I}} \eta_{i j}(\vartheta)\left(A_{i}^{\prime}\right)^{\vartheta} \mathcal{P}_{j}(\vartheta) A_{i}^{\vartheta}-\mathcal{P}_{i}(\sigma) \prec 0
$$

where $\eta_{i j}(\vartheta) \triangleq \pi_{i j}(\vartheta) / \rho_{i}(\vartheta)$ is defined in (15).

Proof. Consider the PDF of sojourn time that is subject to the EMP distribution:

$$
\omega_{i j}(t+K T)=\omega_{i j}(t) \mu_{i j}^{K T}, \forall i \neq j, i, j \in \mathcal{I} .
$$

where $0 \leq \omega_{i j}(t) \leq 1,0<\mu_{i j}<1, t \in \mathbb{N}_{+}$. Without loss of generality, we assume that $r_{k_{n}}=i, r_{k_{n+1}}=j, r_{k_{n+2}}=p, i \neq$ $j, j \neq p, i, j, p \in \mathcal{I}$ and that $S_{n+1}=K_{n+1} T+\tau_{n+1}, S_{n+2}=$ $K_{n+2} T+\tau_{n+2}, K_{n+1}, K_{n+2} \in \mathbb{N}, \tau_{n}, \tau_{n+1}, \tau_{n+2} \in[1, T]$. Then, for $n, n+1$, it can be obtained from (15) that

$$
\begin{array}{r}
\sum_{j \neq i, j \in \mathcal{I}}\left[\frac{\theta_{i j}}{\rho_{i}\left(S_{n+1}\right)} w_{i j}\left(S_{n+1}\right)\left(A_{i}^{\prime}\right)^{S_{n+1}} P_{j}\left(n+1, S_{n+1}\right)\right. \\
\left.\times A_{i}^{S_{n+1}}\right]-P_{i}\left(n, S_{n}\right) \triangleq-Q_{i}\left(n, S_{n}\right)
\end{array}
$$

where $Q_{i}\left(n, S_{n}\right) \succ 0$. Due to $S_{n+1}=K_{n+1} T+\tau_{n+1}$, is equivalent to

$$
\begin{aligned}
& \sum_{j \neq i, j \in \mathcal{I}}\left[\frac{\theta_{i j}}{\rho_{i}\left(\tau_{n+1}\right)} w_{i j}\left(\tau_{n+1}\right)\left(A_{i}^{\prime}\right)^{\tau_{n+1}} \frac{\rho_{i}\left(\tau_{n+1}\right)}{\rho_{i}\left(S_{n+1}\right)}\left(\mu_{i j}\right)^{K_{n+1} T}\right. \\
& \left.\left(A_{i}^{\prime}\right)^{K_{n+1} T} P_{j}\left(n+1, S_{n+1}\right) A_{i}^{K_{n+1} T} A_{i}^{\tau_{n+1}}\right]-P_{i}\left(n, S_{n}\right) \\
= & -Q_{i}\left(n, S_{n}\right)
\end{aligned}
$$

Assuming that $\vartheta=\tau_{n+1}, \sigma=\tau_{n}$, it can be obtained from (20) that

$$
\begin{array}{r}
\sum_{j \neq i, j \in \mathcal{I}} \frac{\theta_{i j}}{\rho_{i}\left(\tau_{n+1}\right)} w_{i j}\left(\tau_{n+1}\right)\left(A_{i}^{\prime}\right)^{\tau_{n+1}} \mathcal{P}_{j}\left(\tau_{n+1}\right) A_{i}^{\tau_{n+1}} \\
-\mathcal{P}_{i}\left(\tau_{n}\right)=-\mathcal{Q}_{i}\left(\tau_{n}\right)
\end{array}
$$

Comparing (23) and (24), one can observe that if we set

$$
\begin{aligned}
& P_{j}\left(n+1, S_{n+1}\right) \\
= & \frac{\rho_{i}\left(S_{n+1}\right)}{\rho_{i}\left(\tau_{n+1}\right)}\left(\mu_{i j}\right)^{-K_{n+1} T}\left(A_{i}^{\prime}\right)^{-K_{n+1} T} \mathcal{P}_{j}\left(\tau_{n+1}\right) A_{i}^{-K_{n+1} T} \\
& P_{i}\left(n, S_{n}\right)=\mathcal{P}_{i}\left(\tau_{n}\right), Q_{i}\left(n, S_{n}\right)=\mathcal{Q}_{i}\left(\tau_{n}\right)
\end{aligned}
$$

then, (23) holds if and only if (24) holds. Similarly, for $n+$ $1, n+2$, the following equation can be derived from (15):

$$
\begin{array}{r}
\sum_{p \neq j, p \in \mathcal{I}} \frac{\theta_{j p}}{\rho_{j}\left(S_{n+2}\right)} w_{j p}\left(S_{n+2}\right)\left(A_{j}^{\prime}\right)^{S_{n+2}} P_{p}\left(n+2, S_{n+2}\right) A_{j}^{S_{n+2}} \\
-P_{j}\left(n+1, S_{n+1}\right)=-Q_{j}\left(n+1, S_{n+1}\right)(27)
\end{array}
$$

Substituting (25) into (27), one has

$$
\begin{aligned}
& \sum_{p \neq j, p \in \mathcal{I}}\left[\frac{\theta_{j p}}{\rho_{j}\left(S_{n+2}\right)} w_{j p}\left(S_{n+2}\right)\left(A_{j}^{\prime}\right)^{S_{n+2}} P_{p}\left(n+2, S_{n+2}\right)\right. \\
& \left.\times\left(A_{j}\right)^{S_{n+2}}\right]-\frac{\rho_{i}\left(S_{n+1}\right)}{\rho_{i}\left(\tau_{n+1}\right)}\left(\mu_{i j}\right)^{-K_{n+1} T}\left(A_{i}^{\prime}\right)^{-K_{n+1} T} \\
& \times \mathcal{P}_{j}\left(\tau_{n+1}\right) A_{i}^{-K_{n+1} T}=-Q_{j}\left(n+1, S_{n+1}\right) \\
\Leftrightarrow & \sum_{p \neq j, p \in \mathcal{I}} \frac{\theta_{j p}}{\rho_{j}\left(\tau_{n+2}\right)} w_{j p}\left(\tau_{n+2}\right)\left(A_{j}^{\prime}\right)^{\tau_{n+2}}\left[\frac{\rho_{j}\left(\tau_{n+2}\right) \rho_{i}\left(\tau_{n+1}\right)}{\rho_{j}\left(S_{n+2}\right) \rho_{i}\left(S_{n+1}\right)}\right. \\
& \times\left(\mu_{j p}\right)^{K_{n+2} T}\left(A_{j}^{\prime}\right)^{-\tau_{n+2}}\left(\mu_{i j}\right)^{K_{n+1} T}\left(A_{i}^{\prime}\right)^{K_{n+1} T}\left(A_{j}^{\prime}\right)^{S_{n+2}} \\
& \left.\times P_{p}\left(n+2, S_{n+2}\right) A_{j}^{S_{n+2}} A_{i}^{K_{n+1} T} A_{j}^{-\tau_{n+2}}\right] A_{j}^{\tau_{n+2}} \\
& -\mathcal{P}_{j}\left(\tau_{n+1}\right)=-\frac{\rho_{i}\left(\tau_{n+1}\right)}{\rho_{i}\left(S_{n+1}\right)}\left(\mu_{i j}\right)^{K_{n+1} T}\left(A_{i}^{\prime}\right)^{K_{n+1} T} \\
& \times Q_{j}\left(n+1, S_{n+1}\right) A_{i}^{K_{n+1} T}
\end{aligned}
$$

Correspondingly, let $\vartheta=\tau_{n+2}, \sigma=\tau_{n+1}$, and one knows from (20) that

$$
\begin{array}{r}
\sum_{p \neq j, p \in \mathcal{I}} \frac{\theta_{j p}}{\rho_{j}\left(\tau_{n+2}\right)} w_{j p}\left(\tau_{n+2}\right)\left(A_{j}^{\prime}\right)^{\tau_{n+2}} \mathcal{P}_{p}\left(\tau_{n+2}\right) A_{j}^{\tau_{n+2}} \\
-\mathcal{P}_{j}\left(\tau_{n+1}\right)=-\mathcal{Q}_{j}\left(\tau_{n+1}\right) .
\end{array}
$$

Set

$$
\begin{aligned}
& P_{p}\left(n+2, S_{n+2}\right) \\
= & \frac{\rho_{j}\left(S_{n+2}\right) \rho_{i}\left(S_{n+1}\right)}{\rho_{j}\left(\tau_{n+2}\right) \rho_{i}\left(\tau_{n+1}\right)}\left(\mu_{j p}\right)^{-K_{n+2} T}\left(\mu_{i j}\right)^{-K_{n+1} T}\left(A_{j}^{\prime}\right)^{-S_{n+2}} \\
& \times\left(A_{i}^{\prime}\right)^{-K_{n+1} T}\left(A_{j}^{\prime}\right)^{\tau_{n+2}} \mathcal{P}_{p}\left(\tau_{n+2}\right) A_{j}^{\tau_{n+2}} A_{i}^{-K_{n+1} T} A_{j}^{-S_{n+2}}
\end{aligned}
$$$$
Q_{j}\left(n+1, S_{n+1}\right)
$$$$
=\frac{\rho_{i}\left(S_{n+1}\right)}{\rho_{i}\left(\tau_{n+1}\right)}\left(\mu_{i j}\right)^{-K_{n+1} T}\left(A_{i}^{\prime}\right)^{-K_{n+1} T} \mathcal{Q}_{p}\left(\tau_{n+2}\right) A_{i}^{-K_{n+1} T}
$$

Then, (28) is equivalent to (29). Iterating the above process, it is straightforward that for any $k_{m}, S_{m}=\tau_{m}+K_{m} T, r_{k_{m}}=$ $q, m>n+2 \in \mathbb{N}_{+}, \tau_{m} \in[1, T], K_{m} \in \mathbb{N}, q \in \mathcal{I}$, there will be a set of $P_{q}\left(m, S_{m}\right)$ and a set of $Q_{q}\left(m, S_{m}\right)$ that can be obtained by $\mathcal{P}_{q}\left(\tau_{m}\right)$ and $\mathcal{Q}_{q}\left(\tau_{m}\right)$. Therefore, for any given sequence $(n, i, \alpha)$, one can always find a set of positive matrices $P_{i}(n, \alpha)$ such that (15) holds if and only if (20) holds. This completes the proof.

Derived from Theorem 2 (i), Theorem 4 gives the stability criterion that consists of a finite number of inequalities when the the PDF of sojourn time is considered to be EMP. In a same vein to Theorem 2(ii), the following corollary presents another form of stability criterion and the proof is omitted here.

Corollary 2: Consider S-MJLS (1) with $u(k) \equiv 0$ and the sojourn time being subject to a set of EMP distributions with the same period $T$ for each system mode. Then, system (1) is MSS if there exists a set of symmetric matrices $O_{i}(\sigma, m) \succ 0$, $i \in \mathcal{I}, \sigma \in \mathbb{N}_{[1, T]}, m \leq \sigma, m \in \mathbb{N}_{[0, T]}$ such that the following inequalities hold for all $j \neq i, i, j \in \mathcal{I}, \sigma, \vartheta \in \mathbb{N}_{[1, T]}$, and $m<\vartheta, m \in \mathbb{N}_{[0, T]}$

$$
\sum_{j \neq i, j \in \mathcal{I}} \eta_{i j}(\vartheta)\left\{A_{i}^{\prime} O_{j}(\vartheta, m+1) A_{i}-O_{j}(\vartheta, m)\right\} \prec 0
$$




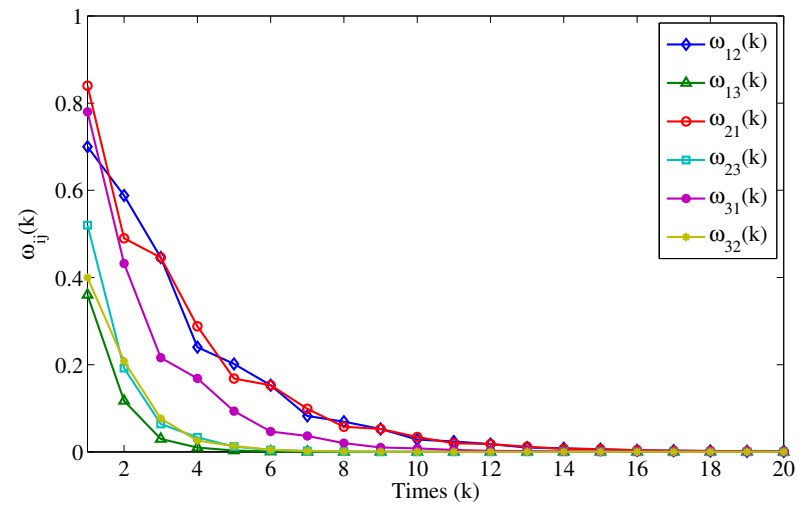

(a) The underlying EMP distributions $\omega_{i j}(k)$ with $T=3$.

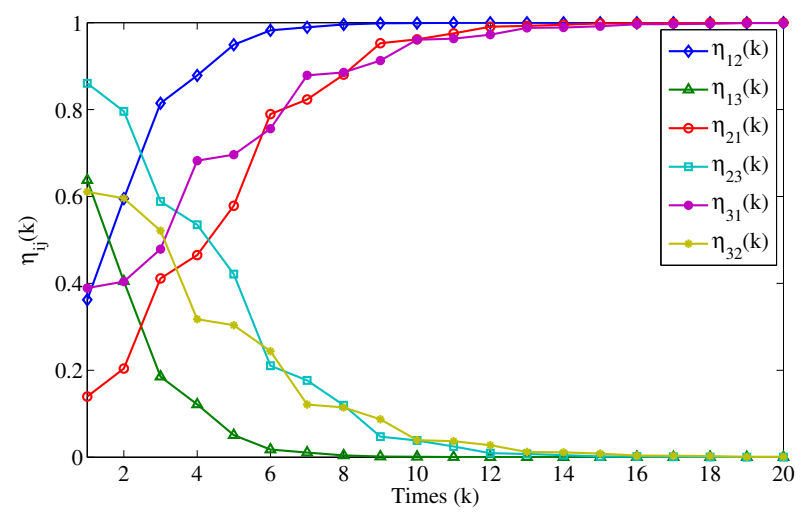

(c) The underlying $\eta_{i j}(k)$ with $T=3$.

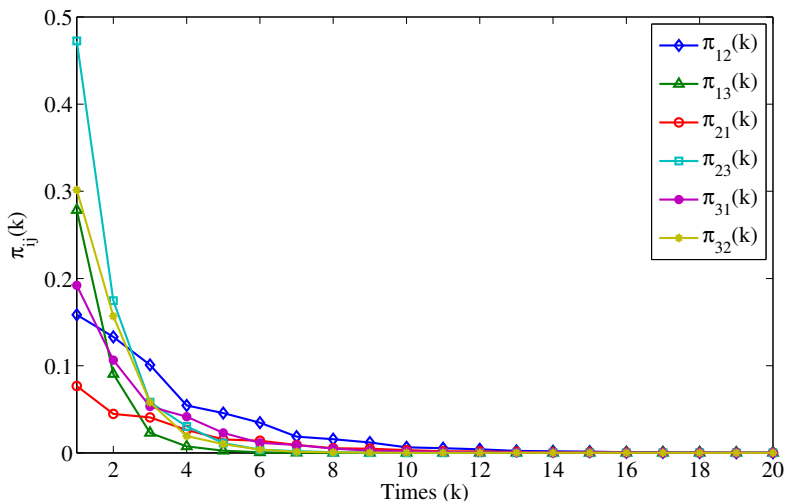

(b) The underlying $\pi_{i j}(k)$ with $T=3$.

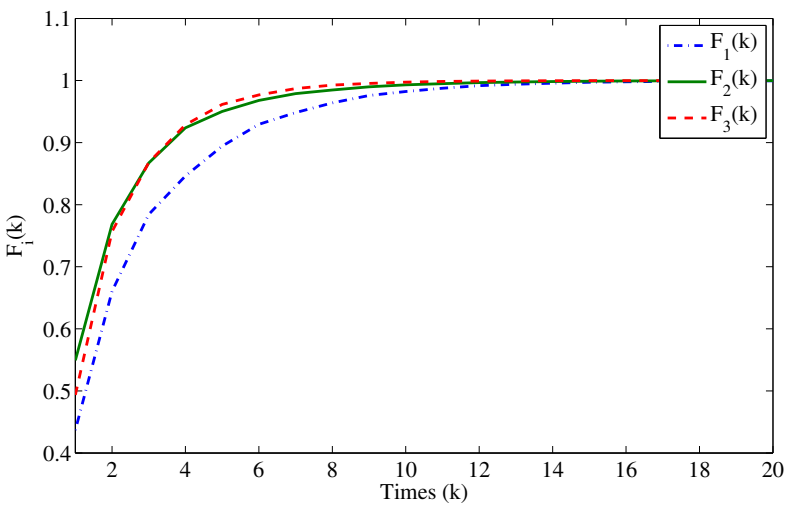

(d) The underlying $F_{i}(k)$ with $T=3$.

Fig. 3: Illustration of the PDF subject to EMP distributions and the corresponding probabilities in Example 1.

$$
\sum_{j \neq i, j \in \mathcal{I}} \eta_{i j}(\vartheta) O_{j}(\vartheta, 0)-O_{i}(\sigma, \sigma) \prec 0
$$

where $\eta_{i j}(\vartheta)$ is given in (15).

Based on Corollary 2, the stabilization criterion in form of a set of linear matrix inequalities can be developed as follows (the proof is also omitted here, which can be readily done by referring to the proof for Theorem 3).

Corollary 3: Consider S-MJLS (1) with the controller in the form of (5) and the sojourn time being subject to a set of EMP distributions with the same period $T$ for each system mode. Then, system (1) is MSS if there exist a set of matrices $Z, U_{i}(\sigma)$, and a set of symmetric matrices $H_{i}(\sigma, m) \succ 0$, $i \in \mathcal{I}, \sigma \in \mathbb{N}_{[1, T]}, m \leq \sigma, m \in \mathbb{N}_{[0, T]}$ such that the following inequalities hold for all $i \neq j, i, j \in \mathcal{I}, \sigma, \vartheta \in \mathbb{N}_{[1, T]}, m<\vartheta$, $m \in \mathbb{N}_{[0, T]}$

$$
\begin{array}{r}
{\left[\begin{array}{cc}
\widetilde{\mathcal{H}}_{i}(\vartheta, m+1)-\widetilde{\mathcal{Z}}-\widetilde{\mathcal{Z}}^{\prime} & \check{\mathcal{A}}_{i}(\sigma) \widetilde{\mathcal{L}}_{i}(\vartheta) \\
* & -\widetilde{\mathcal{L}}_{i}^{\prime}(\vartheta) \widetilde{\mathcal{H}}_{i}(\vartheta, m) \widetilde{\mathcal{L}}_{i}(\vartheta)
\end{array}\right] \prec 0} \\
{\left[\begin{array}{cc}
\widetilde{\mathcal{H}}_{i}(\vartheta, 0)-\widetilde{\mathcal{Z}}-\widetilde{\mathcal{Z}}^{\prime} & \widetilde{\mathcal{L}}_{i}(\vartheta) Z \\
* & -H_{i}(\sigma, \sigma)
\end{array}\right] \prec 0}
\end{array}
$$

where $\widetilde{\mathcal{H}}_{i}(\vartheta, m) \triangleq \operatorname{diag}\left\{H_{1}(\vartheta, m), \ldots, H_{i-1}(\vartheta, m), H_{i+1}(\vartheta\right.$, $\left.m), \ldots, H_{M}(\vartheta, m)\right\}, \widetilde{\mathcal{Z}} \triangleq \operatorname{diag}_{(M-1)}\{Z\}, \tilde{\mathcal{A}}_{i}(\sigma) \triangleq$ $\operatorname{diag}_{(M-1)}\left\{A_{i} Z+B_{i} U_{i}(\sigma)\right\}, \quad \widetilde{\mathcal{L}}_{i}(\vartheta) \triangleq\left[\sqrt{\eta_{i 1}(\vartheta)} \mathbf{I}, \ldots\right.$, $\left.\sqrt{\eta_{i(i-1)}(\vartheta)} \mathbf{I}, \sqrt{\eta_{i(i+1)}(\vartheta)} \mathbf{I}, \ldots, \sqrt{\eta_{i M}(\vartheta)} \mathbf{I}\right]^{\prime}$, and $\eta_{i j}(\vartheta)$ is defined in (15). Moreover, the admissible controller gain for (5) is given by

$$
K_{i}(\sigma)=U_{i}(\sigma) Z^{-1} .
$$

Remark 6: According to Remark 3, one can observe that the semi-Markov processes with geometric PDF of sojourn time will reduce to Markov processes. However, it is worth noting that the above-derived criteria based on the horizon of jumping instants $k_{n}$ cannot directly reduce to the commonly existing MSS stability criteria for Markov jump systems that base the derivations on the horizon of sampling instants $k$ due to the benefit of memoryless TPs.

Remark 7: Another noteworthy observation is that, in either case of general PDF of sojourn time and EMP PDF of sojourn time, all the controller gains given by Theorem 1, Theorem 3 or Corollary 3 are STD. A direct question can be raised that in the case of general PDF of sojourn time, via a sojourn-timeindependent (STI) Lyapunov function, one may obtain a STI controller gain $K_{i}$ as usually obtained in the context of MJLSs, which seems to be of limited number of computations as $K_{i}$ is independent of sojourn time. However, notice that the general PDF $\omega_{i j}(k)$ of sojourn time will still be contained in the resulting criterion, and it remains unchanged that an infinite number of conditions need to be checked (determined by the memory TPs in essence). Meanwhile, such a criterion will be 


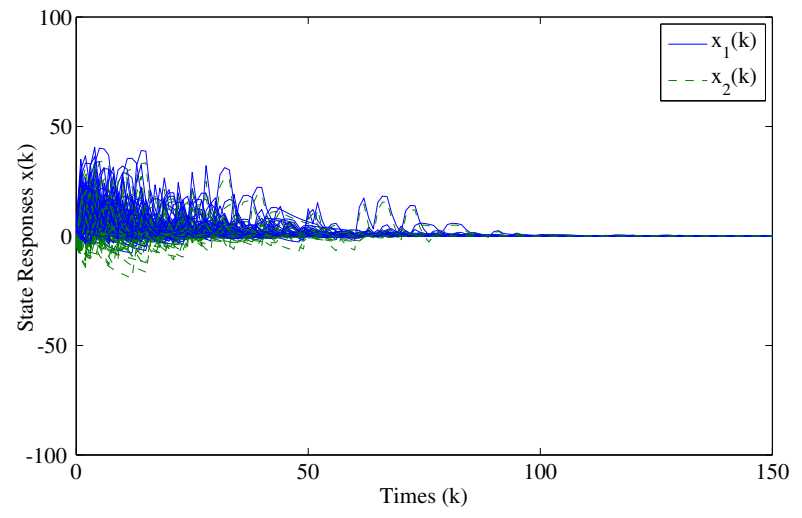

(a) $\alpha=0.70$

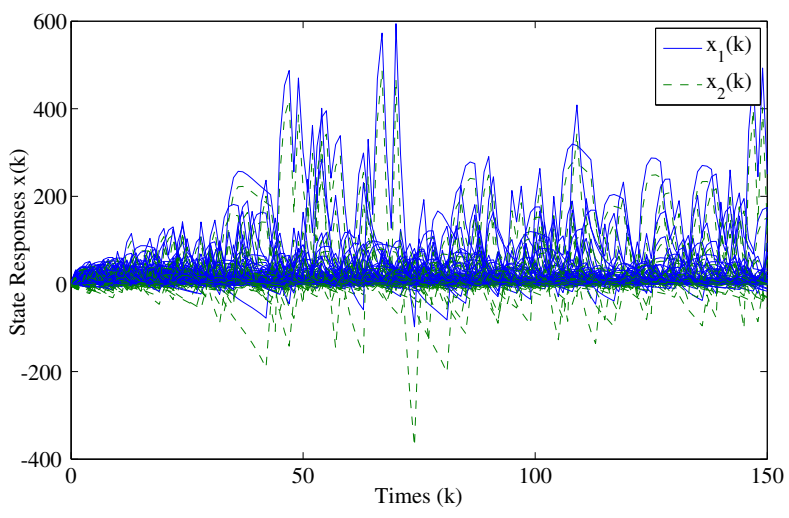

(b) $\alpha=0.75$

Fig. 4: 100 realizations of state responses for different random jumping sequences for $\alpha=0.70$ and $\alpha=0.75$.

rather conservative since it is derived from a stability criterion that will be only sufficient by using a STI Lyapunov function (with less freedom). The conservatism will be inherited in the case of EMP PDF of sojourn time. On the contrary, the STD Lyapunov function used in the paper yields STD controller (i.e., offers more freedom in finding an admissible stabilizing controller than STI method) and as such is less conservative.

\section{Illustrative EXAMPLES}

In this section, a numerical example and an application in a class of population ecological systems are provided to demonstrate the validity and applicability of the obtained theoretical results.

\section{A. Example 1 (numerical example)}

Consider a S-MJLS with three modes with system matrices

$$
\begin{aligned}
& A_{1}=\alpha\left[\begin{array}{rr}
1.55 & -0.85 \\
0.50 & 0.15
\end{array}\right], B_{1}=\left[\begin{array}{r}
-0.1 \\
0.1
\end{array}\right] \\
& A_{2}=\alpha\left[\begin{array}{rr}
5.41 & -4.73 \\
4.27 & -3.61
\end{array}\right], B_{2}=\left[\begin{array}{c}
0.1 \\
0.1
\end{array}\right] \\
& A_{3}=\alpha\left[\begin{array}{rr}
0.27 & 0.50 \\
-1.31 & 2.03
\end{array}\right], B_{3}=\left[\begin{array}{c}
0.1 \\
-0.1
\end{array}\right]
\end{aligned}
$$

where $\alpha>0$ is a constant parameter that can characterize the distance between system eigenvalues and unit circle. The switching among the three modes is governed by a SMC, where the PDF of sojourn time are given by the following EMP distribution

$$
\omega_{i j}(k)=\left\{\begin{array}{cc}
\beta_{i j}(1) \cdot \mu_{i j}^{K T}, & k=K T+1, K \in \mathbb{N} \\
\beta_{i j}(2) \cdot \mu_{i j}^{K T}, & k=K T+2, K \in \mathbb{N} \\
\cdots & \\
\beta_{i j}(T) \cdot \mu_{i j}^{K T}, & k=K T+T, K \in \mathbb{N}
\end{array}\right.
$$

where $i, j \in \mathcal{I}$. Considering $T=3$ and defining $\beta_{i j} \triangleq$ $\left[\begin{array}{lll}\beta_{i j}(1) & \beta_{i j}(2) & \beta_{i j}(3)\end{array}\right]^{\prime}$, we set $\beta_{12}=\left[\begin{array}{llll}0.70 & 0.84 & 0.91\end{array}\right]^{\prime}$, $\beta_{13}=\left[\begin{array}{lll}0.36 & 0.39 & 0.33\end{array}\right]^{\prime}, \quad \beta_{21}=\left[\begin{array}{llll}0.87 & 0.70 & 0.91\end{array}\right]^{\prime}$, $\beta_{23}=\left[\begin{array}{lll}0.52 & 0.48 & 0.40\end{array}\right]^{\prime}, \beta_{31}=\left[\begin{array}{lll}0.78 & 0.72 & 0.60\end{array}\right]^{\prime}, \beta_{32}=$ $\left[\begin{array}{lll}0.40 & 0.52 & 0.48\end{array}\right]^{\prime}, \mu_{12}=0.7, \mu_{13}=0.3, \mu_{21}=0.7, \mu_{23}=0.4$, $\mu_{31}=0.6$, and $\mu_{32}=0.4$. The illustrations of the above EMP distribution $w_{i j}(k)$ are shown in Fig. 3. The TPs $\theta_{i j}$ are given as

$$
\Theta=\left[\begin{array}{ccc}
0 & 0.2262 & 0.7738 \\
0.0912 & 0 & 0.9088 \\
0.2463 & 0.7537 & 0
\end{array}\right]
$$

It can be checked that $\sum_{k \in \mathbb{N}_{+}} \sum_{j \neq i, j \in \mathcal{I}} \theta_{i j} \omega_{i j}(k)=1$.

First, the stability of unforced system can be testified by Corollary 2. Given different $\alpha$, Table I lists the computation results whether a feasible solution to Corollary 2 exists, and the simulation results (state responses of the systems when randomly generating different switching sequences). In the simulation, the considered initial system states satisfy $x_{1} \in[0,5], x_{2} \in[-5,0]$ and the initial system mode is subject to $\operatorname{Pr}\left(r_{0}=1\right)=0.3, \operatorname{Pr}\left(r_{0}=2\right)=0.3, \operatorname{Pr}\left(r_{0}=3\right)=0.4$. The two cases $\alpha=0.70$ and $\alpha=0.75$ are given in Fig. 4, where 100 realizations of state responses are presented. As seen from both Table I and Fig. 4, the system is checked to be MSS for $\alpha \geq 0.65$ based on Corollary 2, and is shown to be stable for $\alpha=0.70$ and unstable for $\alpha=0.75$ in the simulation. Therefore, it can be verified that, despite the fact that only sufficient conditions are obtained, Corollary 2 is effective in judging the stability of the system.

Then, by Corollary 3, the desired mode-dependent STD controller can be designed such that the resulting closed-loop system is MSS. For $\alpha=1$, the corresponding controller gains are given as below

$$
\begin{aligned}
& K_{1}(1)=\left[\begin{array}{ll}
5.7333 & -5.1649
\end{array}\right], K_{1}(2)=\left[\begin{array}{ll}
5.7085 & -5.1431
\end{array}\right] \\
& K_{1}(3)=\left[\begin{array}{ll}
5.6737 & -5.1172
\end{array}\right], K_{2}(1)=\left[\begin{array}{ll}
-76.9445 & 69.7061
\end{array}\right] \\
& K_{2}(2)=\left[\begin{array}{ll}
-78.3732 & 71.0986
\end{array}\right], K_{2}(3)=\left[\begin{array}{ll}
-83.1822 & 74.8398
\end{array}\right] \\
& K_{3}(1)=\left[\begin{array}{ll}
-7.6986 & 7.1717
\end{array}\right], K_{3}(2)=\left[\begin{array}{ll}
-7.2521 & 6.7413
\end{array}\right] \\
& K_{3}(3)=\left[\begin{array}{ll}
-7.5194 & 6.9280
\end{array}\right]
\end{aligned}
$$

TABLE I: Verification of the validity of Corollary 2

\begin{tabular}{ccc}
\hline$\alpha$ & Corollary 2 & Simulation \\
\hline 0.60 & Feasible & Stable \\
0.65 & Feasible & Stable \\
0.70 & Infeasible & Stable \\
0.75 & Infeasible & Unstable \\
\hline
\end{tabular}




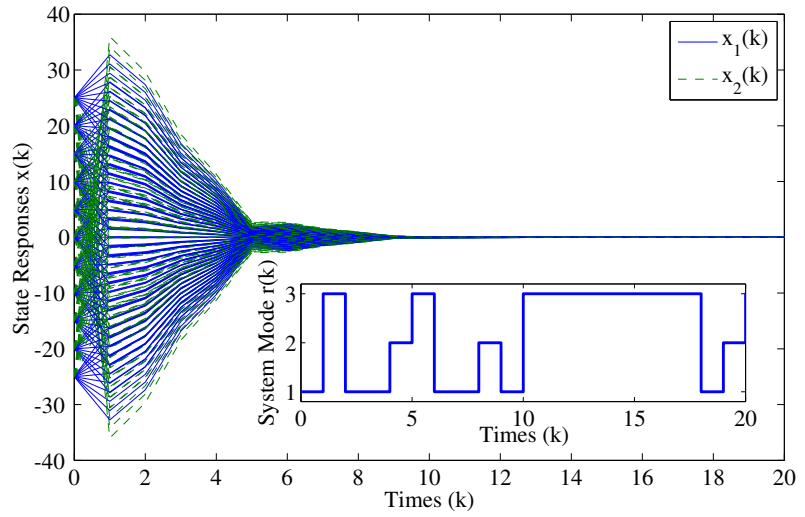

(a) First set of state responses

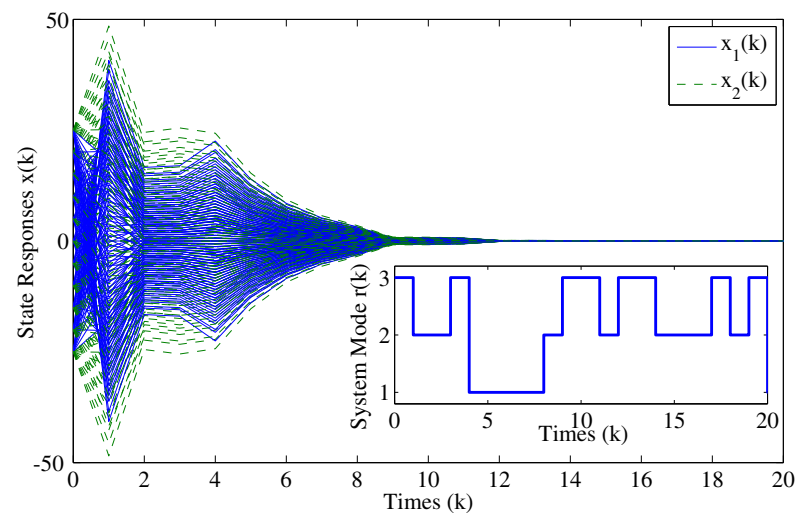

(c) Second set of state responses

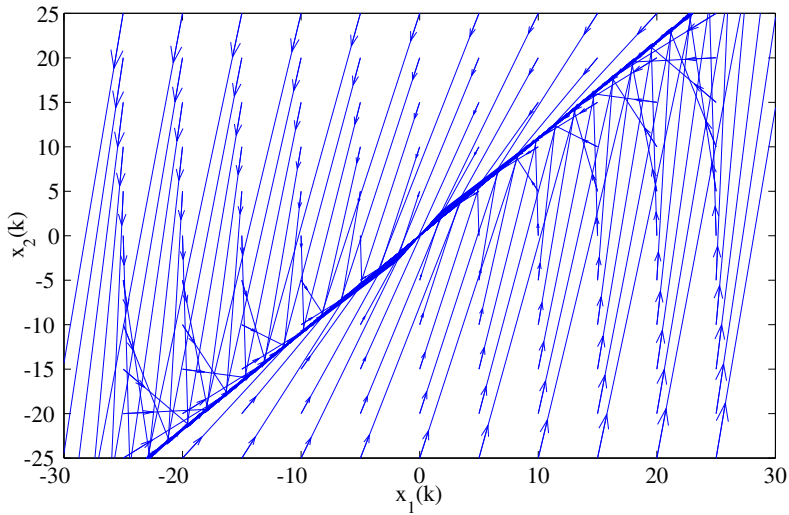

(b) Phase diagram for the first set of state responses

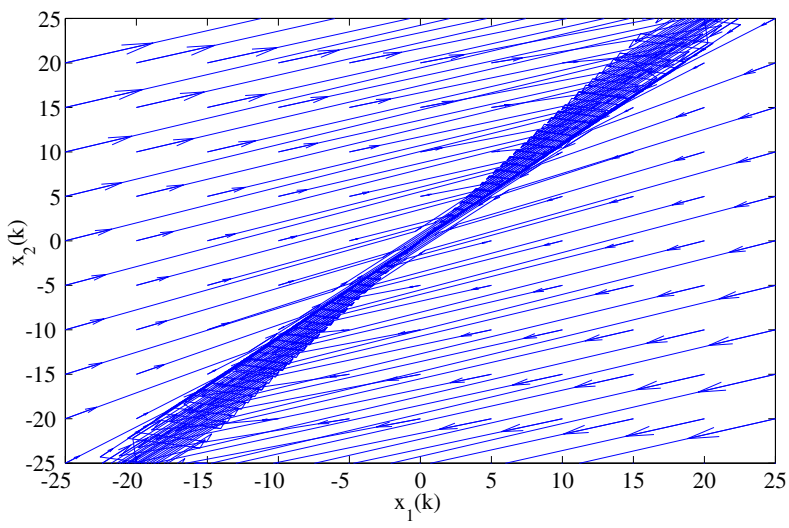

(d) Phase diagram for the second set of state responses

Fig. 5: Two sets of 100 realizations of state responses for different initial states $x(k)$ for $\alpha=1$ with control gain $K_{i}(\sigma)$ and the corresponding phase diagrams.

Based on the derived controller gain $K_{i}(\sigma), i \in \mathcal{I}, \sigma \in$ $[1, T]$, two sets of 100 realizations of the state responses of the closed-loop system for 100 different initial conditions $x_{0}=\left[\begin{array}{ll}x_{1}(0) & x_{2}(0)\end{array}\right]^{\prime}$ where $x_{1}(0), x_{2}(0) \in\left[\begin{array}{ll}-25 & 25\end{array}\right]$ are shown in Fig. 5. The used two switching sequences are stochastically produced based on the same PDF distribution, $\Theta$, and initial system mode probabilities. It can be observed from Fig. 5 that the controller designed by Corollary 3 is valid.

\section{B. Example 2 (illustrative example of a class of population ecological systems)}

Consider an ecological system consisting of four types of population $P_{1}, P_{2}, P_{3}, P_{4}$ and let the number of individuals in population $P_{i}, i=1,2,3,4$ be denoted by $N_{1}, N_{2}, N_{3}$, and $N_{4}$, respectively. Suppose the situated environment be subject to stochastic autonomous switching among three scenarios $\mathbb{E}_{1}$, $\mathbb{E}_{2}$, and $\mathbb{E}_{3}$. The basic mathematical principle of growth of $N_{i}, i=1,2,3,4$ obeys the following logistic equation [27]

$$
\dot{N}_{i}=a_{i}^{(\sigma)} N_{i}\left(1-\frac{N_{i}}{K_{i}^{(\sigma)}}\right)
$$

where $a_{i}^{(\sigma)}$ is the maximum per-capita rate of change of $P_{i}$, and $K_{i}^{(\sigma)}$ is the carrying capacity of the population in environment $\mathbb{E}_{\sigma}, \sigma=1,2,3$. In essence, the dynamics described for the variation of $N_{i}$ in (37) is nonlinear. Assume $K_{i}^{(\sigma)}$ to be approximately proportional to $N_{i}$, i.e., $K_{i}^{\sigma}=\left(1 / \rho_{i}^{(\sigma)}\right) N_{i}$, and include the mutual influence among four types of population and the effect of external intervention $u_{i}$ (intentionally immigrates or hunts some individuals) on $N_{i}$ [28], the equation (37) is extended as

$$
\dot{N}_{i}=a_{i}^{(\sigma)} N_{i}\left(1-\rho_{i}^{(\sigma)}\right)+\sum_{j \neq i}^{4} b_{i j}^{(\sigma)} N_{j}+c_{i}^{(\sigma)} u_{i}
$$

where $b_{i j}^{(\sigma)}$ is a transfer coefficient modeling the mutual influence of $N_{i}$ and $N_{j}$, and $c_{i}^{(\sigma)}$ is a transfer coefficient modeling the effect of external intervention on $N_{i}$ in different $\mathbb{E}_{\sigma}$. Letting $x=\left[\begin{array}{llll}N_{1} & N_{2} & N_{3} & N_{4}\end{array}\right]^{\prime}$ and $u=\left[\begin{array}{llll}u_{1} & u_{2} & u_{3} & u_{4}\end{array}\right]^{\prime}$ denote the system states and control input, respectively, the discrete-time model of (38) can be obtained by the first-order Euler approximation approach with sampling time $T_{s}$ :

$$
x(k+1)=A^{(\sigma)} x(k)+B^{(\sigma)} u(k)
$$

where

$$
A^{(\sigma)}=\left[\begin{array}{cccc}
\hat{a}_{1} & b_{12}^{(\sigma)} & b_{13}^{(\sigma)} & b_{14}^{(\sigma)} \\
b_{21}^{(\sigma)} & \hat{a}_{2} & b_{23}^{(\sigma)} & b_{24}^{(\sigma)} \\
b_{31}^{(\sigma)} & b_{32}^{(\sigma)} & \hat{a}_{3} & b_{34}^{(\sigma)} \\
b_{41}^{(\sigma)} & b_{42}^{(\sigma)} & b_{43}^{(\sigma)} & \hat{a}_{4}
\end{array}\right] T_{s}+\mathbf{I},
$$




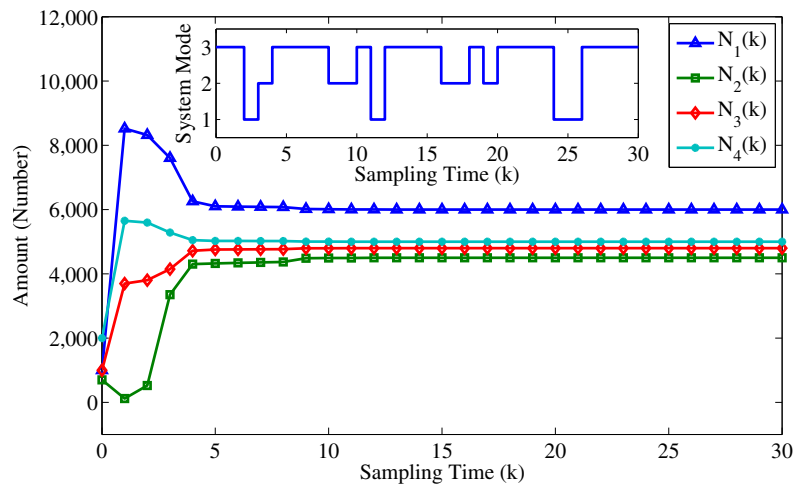

(a) State response and system mode sequence for Case 1

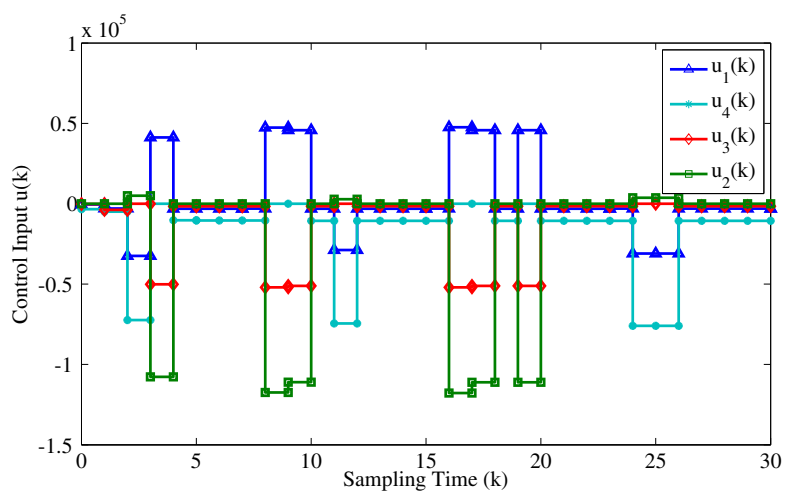

(c) Control input for Case 1

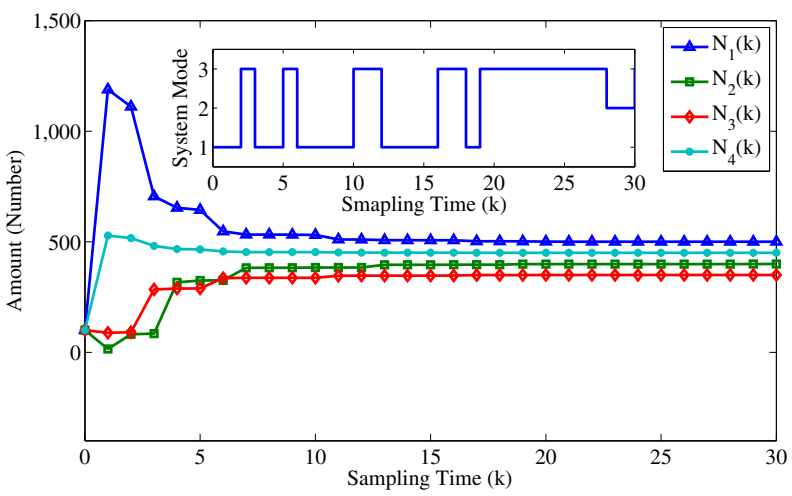

(b) State response and system mode sequence for Case 2

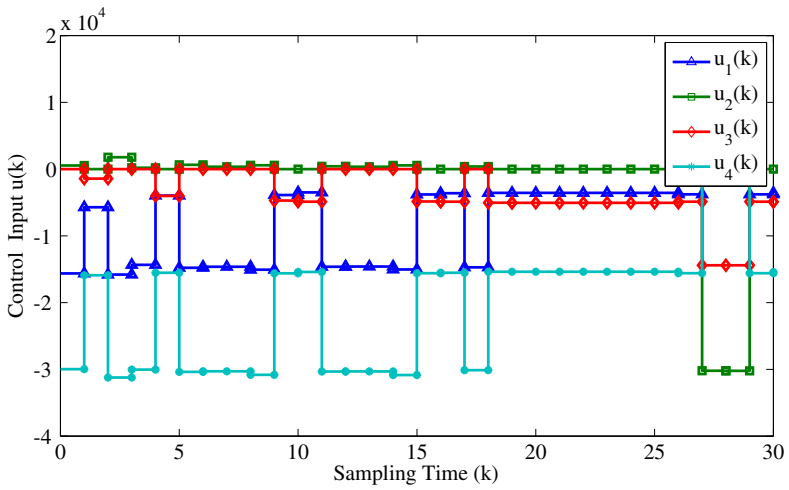

(d) Control input for Case 2

Fig. 6: State responses and control inputs for the four types of population for Cases 1 and 2.

TABLE II: Parameters $\left(a_{i}^{(\sigma)}, \rho_{i}^{(\sigma)}, b_{i}^{(\sigma)}, c_{i}^{(\sigma)}\right), i=1,2,3,4$ in different scenario $\mathbb{E}_{\sigma}, \sigma=1,2,3$

\begin{tabular}{cccc}
\hline $\mathbb{E}_{\sigma}$ & $\mathbb{E}_{1}$ & $\mathbb{E}_{2}$ & $\mathbb{E}_{3}$ \\
\hline$i=1$ & $(2,0.5,[-0.1,-0.2,0.1], 1)$ & $(5,0.1,[-0.2,-0.3,0.2], 2)$ & $(-2,0.8,[-0.1,-0.1,0.1], 2)$ \\
$i=2$ & $(1,0.6,[0.1,0.2,0.1], 1)$ & $(3,0.3,[0.2,0.4,-0.15], 2)$ & $(-1,0.9,[0.1,0.2,-0.2], 1)$ \\
$i=3$ & $(1,0.4,[0.2,0.3,0.1], 0)$ & $(2,0.2,[-0.15,-0.2,-0.15], 1)$ & $(-1,0.6,[-0.2,-0.35,-0.15], 0.5)$ \\
$i=4$ & $(2,0.5,[-0.1,-0.3,-0.2], 0.2)$ & $(4,0.2,[0.2,0.3,0.15], 0)$ & $(-2,0.7,[0.3,0.5,0.2], 0.3)$ \\
\hline
\end{tabular}

$$
B^{(\sigma)}=\left[\begin{array}{rrrr}
c_{1}^{(\sigma)} & 0 & 0 & 0 \\
0 & c_{2}^{(\sigma)} & 0 & 0 \\
0 & 0 & c_{3}^{(\sigma)} & 0 \\
0 & 0 & 0 & c_{4}^{(\sigma)}
\end{array}\right] T_{s}
$$

with

$$
\hat{a}_{i} \triangleq a_{i}^{(\sigma)}\left(1-\rho_{i}^{(\sigma)}\right), i=1,2,3,4
$$

where $\left(a_{i}^{(\sigma)}, \rho_{i}^{(\sigma)}, b_{i}^{(\sigma)}, c_{i}^{(\sigma)}\right), i=1,2,3,4$ with $b_{i}^{(\sigma)} \triangleq$ $\left[b_{i j}^{(\sigma)}, b_{i p}^{(\sigma)}, b_{i q}^{(\sigma)}\right], j, p, q \in\{1,2,3,4\}, j<p<q, j \neq p \neq$ $q \neq i$ is given in Table II.

The purpose is to regulate $N_{1}, N_{2}, N_{3}$, and $N_{4}$ to the pre-set equilibrium $N^{*}=\left[N_{1}^{*}, N_{2}^{*}, N_{3}^{*}, N_{4}^{*}\right]^{\prime}$ against the autonomous variation of $E_{\sigma}$. Two different types of PDF of sojourn time are considered. The first one (Case 1) is the same as in (36) in Example 1. For the second one (Case 2), we would like to take the first three typical distributions in Fig. 1(a) and consider an approximation with $T=3$. We reset (36) with $\beta_{12}(k)=0.2(1-0.2)^{(k-1)}, \beta_{13}(k)=0.85^{(k-1)^{2}}-$ $0.85^{k^{2}}, \beta_{21}(k)=0.8^{(k-1)^{2}}-0.8^{k^{2}}, \beta_{23}(k)=0.3^{k}(1-$ $0.3)^{(15-k)} 15 ! /((15-k) ! i !), \beta_{31}(k)=0.45^{(k-1)^{2}}-0.45^{k^{2}}$, $\beta_{32}(k)=0.7^{k}(1-0.7)^{(15-k)} 15 ! /((15-k) ! i !)$ for $k=1,2,3$, and the parameters $\mu_{i j}, i, j \in\{1,2,3\}$ are the same. The TPs $\theta_{i j}$ for Case 2 are given as

$$
\Theta=\left[\begin{array}{ccc}
0 & 0.0001 & 0.9999 \\
0.1546 & 0 & 0.8454 \\
0.9883 & 0.0117 & 0
\end{array}\right]
$$

We set $T_{s}=1$ (time unit), the pre-set equilibrium $N^{*}=$ $[6000,4500,4800,5000]^{\prime}$, and $T_{s}=0.1$ (time unit), the preset equilibrium $N^{*}=[500,400,350,450]^{\prime}$ for the two cases, respectively. Based on Corollary 3 , the controller can be designed and the control gains are omitted here for brevity. Figure 6 shows the state responses and control inputs for the two cases under randomly generated switching sequences for initial conditions $x(0)=[1000,700,1000,2000]^{\prime}$ and $x(0)=[100,100,100,100]^{\prime}$, respectively. It can be seen that the numbers of the four types of population converge to the pre-set equilibria against the stochastic variations of the situated environment illustrating the validity and applicability of the derived theoretical results. 


\section{CONClusion}

The problems of stability and stabilization for a class of discrete-time S-MJLSs in which the sojourn time is subject to EMP PDF were investigated. The general S-MJLSs, for which a finite number of stability and stabilization conditions are hardly obtained, can be approximated by the studied systems especially when the period is relatively large. Necessary and sufficient criterion for the MSS of the S-MJLSs is first explored, but it is not checkable since an infinite number of conditions are involved. However, the developments lay a foundation to further develop the numerically testable conditions for the systems when the PDF of sojourn time are EMP although the sojourn time can tend to infinity. Instead of using the modes TPs in S-MJLSs, which have been shown hardly computable due to their memory characteristics, the PDF information of sojourn time are directly invoked into the derived conditions. A STD Lyapunov function is used such that the STD controller is designed as well while eliminating the terms of power of matrices in the stability conditions. Future works include the extensions of the developed methodologies to the $H_{\infty}$ control and filtering problems for the underlying systems.

\section{ACKNOWLEDGMENT}

The work was supported in part by National Natural Science Foundation of China (61322301), National Natural Science Foundation of Heilongjiang (F201417, JC2015015), the Fundamental Research Funds for the Central Universities, China HIT.BRETIII.201211, HIT.BRETIV.201306.

\section{APPENDIX}

\section{A. Computation of $\epsilon_{i j}(\vec{k})$}

Given the initial mode probability $\operatorname{Pr}\left(r_{1}=i\right)=\bar{\epsilon}_{i}(0)$, we have $\epsilon_{i j}(\overrightarrow{1})=\operatorname{Pr}\left(r_{2}=j \mid r_{1}=i\right)=\operatorname{Pr}\left(r_{1}=\right.$ $\left.i, r_{2}=j\right) / \operatorname{Pr}\left(r_{1}=i\right)=\bar{\epsilon}_{i}(0) \pi_{i j}(1) / \bar{\epsilon}_{i}(0), j \neq i$ and $\epsilon_{i i}(\overrightarrow{1})=1-\sum_{j \neq i, j \in \mathcal{I}} \epsilon_{i j}(\overrightarrow{1})$. Further, one can infer that $\epsilon_{j p}(\overrightarrow{2})=\operatorname{Pr}\left(r_{3}=p \mid r_{2}=j\right)=\left(\sum_{i \neq j, i \in \mathcal{I}} \operatorname{Pr}\left(r_{1}=i, r_{2}=\right.\right.$ $\left.\left.j, r_{3}=p\right)+\operatorname{Pr}\left(r_{1}=j, r_{2}=j, r_{3}=p\right)\right) / \operatorname{Pr}\left(r_{2}=\right.$ $j)=\left(\sum_{i \neq j, i \in \mathcal{I}} \bar{\epsilon}_{i}(0) \epsilon_{i j}(\overrightarrow{1}) \pi_{j p}(1)+\bar{\epsilon}_{j}(0) \pi_{j p}(2)\right) / \sum_{i \in \mathcal{I}}\left(\bar{\epsilon}_{i}(0)\right.$ $\left.\times \epsilon_{i j}(\overrightarrow{1})\right)$ and $\epsilon_{j j}(\overrightarrow{2})=\operatorname{Pr}\left(r_{3}=j \mid r_{2}=j\right)=1-$ $\sum_{p \neq j, p \in \mathcal{I}} \operatorname{Pr}\left(r_{3}=p \mid r_{2}=j\right)$ with $i \neq j, j \neq p$. Similar to the computations of $\epsilon_{i j}(\overrightarrow{1})$ and $\epsilon_{j p}(\overrightarrow{2}), \epsilon_{i j}(\vec{k}), k>2$ can be obtained by

$$
\epsilon_{i j}(\vec{k})=\frac{\operatorname{Pr}\left(r_{k}=i, r_{k+1}=j\right)}{\operatorname{Pr}\left(r_{k}=i\right)}
$$

where

$$
\begin{aligned}
& \operatorname{Pr}\left(r_{k}=i, r_{k+1}=j\right) \\
= & \bar{\epsilon}_{i}(0) \pi_{i j}(k)+\sum_{k_{p}=1}^{k-1} \sum_{p \neq i, p \in \mathcal{I}} \bar{\epsilon}_{p}(0) \pi_{p i}\left(k_{p}\right) \pi_{i j}\left(k-k_{p}\right) \\
& +\sum_{k_{p}=1}^{k-2} \sum_{k_{q}=1}^{k-1-k_{p}} \sum_{p \neq i, p \in \mathcal{I}} \sum_{q \neq p, q \in \mathcal{I}} \bar{\epsilon}_{p}(0) \pi_{p q}\left(k_{p}\right) \\
& \times \pi_{q i}\left(k_{q}\right) \pi_{i j}\left(k-k_{p}-k_{q}\right)+\cdots \\
& +\underbrace{\sum_{p \neq q, p \in \mathcal{I}} \cdots \sum_{g \neq i, g \in \mathcal{I}}}_{k} \bar{\epsilon}_{p}(0) \underbrace{\pi_{p q}(1) \ldots \pi_{g i}(1)}_{k} \pi_{i j}(1)
\end{aligned}
$$

$$
\begin{aligned}
& \operatorname{Pr}\left(r_{k}=i\right) \\
= & \bar{\epsilon}_{i}(0) \lambda_{i i}(k)+\sum_{k_{p}=1}^{k-1} \sum_{p \neq i, p \in \mathcal{I}} \bar{\epsilon}_{p}(0) \pi_{p i}\left(k_{p}\right) \lambda_{i i}\left(k-k_{p}\right) \\
& +\sum_{k_{p}=1}^{k-2} \sum_{k_{q}=1}^{k-1-k_{p}} \sum_{p \neq i, p \in \mathcal{I}} \sum_{q \neq p, q \in \mathcal{I}} \bar{\epsilon}_{p}(0) \pi_{p q}\left(k_{p}\right) \\
& \times \pi_{q i}\left(k_{q}\right) \lambda_{i i}\left(k-k_{p}-k_{q}\right)+\cdots \\
& +\underbrace{\sum_{p \neq q, p \in \mathcal{I}} \cdots \sum_{g \neq i, g \in \mathcal{I}}}_{k} \bar{\epsilon}_{p}(0) \underbrace{\pi_{p q}(1) \ldots \pi_{g i}(1)}_{k} \lambda_{i i}(1)
\end{aligned}
$$

Note that $\epsilon_{i j}(\vec{k})$ is dependent on all the possible mode sequences till $k$.

\section{B. Relation Graph of Theorems and Corollaries}

The relationship among the proposed theorems and corollaries are given in Fig. 7.

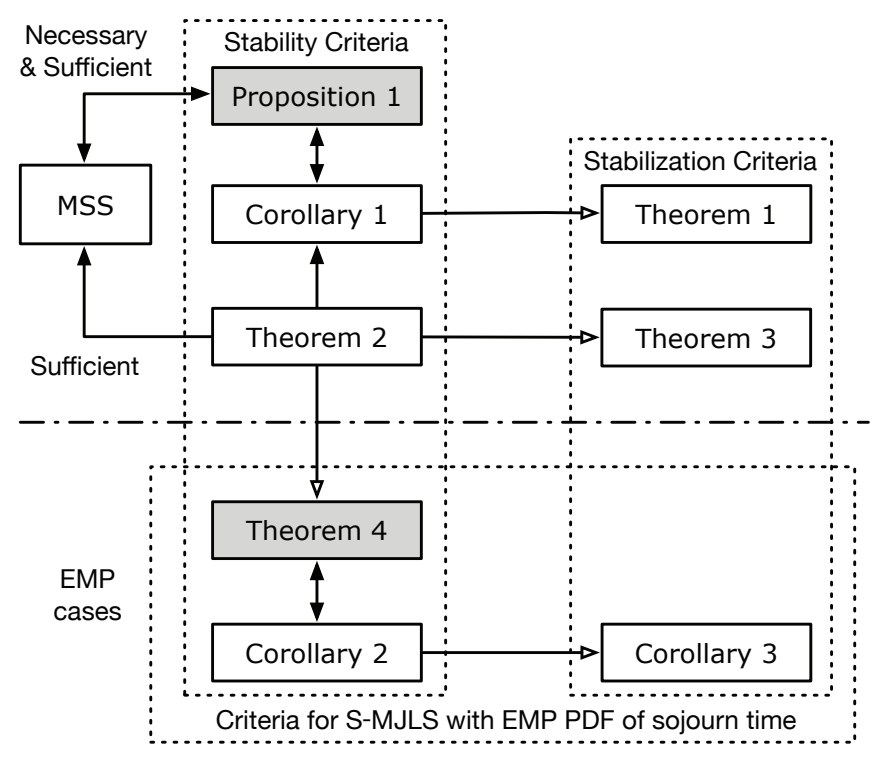

Fig. 7: Relation among the obtained criteria. A solid arrow tells that a theorem/corollary is a sufficient condition to another (the pointed one), and a hollow arrow tells that a criterion (the pointed one) can be derived from another.

\section{Proof of Corollary 1}

Proof. It follows from (11) that

$$
\begin{aligned}
& \sum_{m=0}^{\infty}\left(A_{i}^{\prime}\right)^{m} \sum_{\beta=m+1}^{\infty}\left\{A_{i}^{\prime} \mathcal{O}_{i}(n+1, \beta, m+1) A_{i}\right. \\
& \left.-\mathcal{O}_{i}(n+1, \beta, m)\right\} A_{i}^{m} \\
= & \sum_{\beta=1}^{\infty} \sum_{m=0}^{\beta-1}\left\{\left(A_{i}^{\prime}\right)^{m+1} \mathcal{O}_{i}(n+1, \beta, m+1) A_{i}^{m+1}\right. \\
& \left.-\left(A_{i}^{\prime}\right)^{m} \mathcal{O}_{i}(n+1, \beta, m) A_{i}^{m}\right\} \\
= & \sum_{\beta=1}^{\infty}\left\{\left(A_{i}^{\prime}\right)^{\beta} \mathcal{O}_{i}(n+1, \beta, \beta) A_{i}^{\beta}-\mathcal{O}_{i}(n+1, \beta, 0)\right\} \\
\prec &
\end{aligned}
$$

According to (12), one can further derive that

$$
\sum_{\beta=1}^{\infty}\left\{\left(A_{i}^{\prime}\right)^{\beta} \mathcal{O}_{i}(n+1, \beta, \beta) A_{i}^{\beta}\right\}-O_{i}(n, \alpha, \alpha) \prec 0(40)
$$

By setting $P_{i}(n, \alpha)=O_{i}(n, \alpha, \alpha)$, it can be seen that (7) is equivalent to (40). 


\section{Proof of Theorem 1}

Proof. Letting $\overline{\mathcal{O}}_{i}(n+1, m) \triangleq \sum_{\beta=m+1}^{\infty} \mathcal{O}_{i}(n+1, \beta, m),(11)$ and (12) can be written as, respectively,

$$
\begin{aligned}
A_{i}^{\prime} \mathcal{O}_{i}(n+1, m+1, m+1) A_{i}+A_{i}^{\prime} \overline{\mathcal{O}}_{i}(n+1, m+1) & A_{i} \\
-\overline{\mathcal{O}}_{i}(n+1, m) & \prec 0(41) \\
\overline{\mathcal{O}}_{i}(n+1,0)-O_{i}(n, \alpha, \alpha) & \prec 0(42)
\end{aligned}
$$

From (41), by Schur complement, it yields that

$$
\left[\begin{array}{cc}
-\widehat{\mathcal{O}}_{i}(n+1, m+1, m+1) & 0 \\
* & -\overline{\mathcal{O}}_{i}(n+1, m+1) \\
* & * \\
\widehat{\mathcal{O}}_{i}(n+1, m+1, m+1) \mathcal{A}_{i} \mathcal{L}_{i}(m+1) \\
\overline{\mathcal{O}}_{i}(n+1, m+1) A_{i} \\
-\overline{\mathcal{O}}_{i}(n+1, m)
\end{array}\right]
$$

where $\widehat{\mathcal{O}}_{i}(n, m, m) \triangleq \operatorname{diag}\left\{O_{1}(n, m, m), \ldots, O_{i-1}(n, m, m)\right.$, $\left.O_{i+1}(n, m, m), \ldots, O_{M}(n, m, m)\right\}, \mathcal{A}_{i} \triangleq \operatorname{diag}_{(M-1)}\left\{A_{i}\right\}$. Perform a congruence transformation to (43) by $\operatorname{diag}\left\{\widehat{\mathcal{O}}_{i}^{-1}(n+1, m+1, m+1) \mathcal{V}_{i}, \overline{\mathcal{O}}_{i}^{-1}(n+1, m+1) V_{i}, I\right\}$ with $\mathcal{V}_{i} \triangleq \operatorname{diag}\left\{V_{1}, \ldots, V_{i-1}, V_{i+1}, \ldots, V_{M}\right\}$. Since

$$
\begin{aligned}
\left(\widehat{\mathcal{O}}_{i}(n+1, m+1, m\right. & \left.+1)-\mathcal{V}_{i}\right)^{\prime} \widehat{\mathcal{O}}_{i}^{-1}(n+1, m+1, m+1) \\
& \times\left(\widehat{\mathcal{O}}_{i}(n+1, m+1, m+1)-\mathcal{V}_{i}\right) \succ 0
\end{aligned}
$$

ensures

$$
\begin{aligned}
& \widehat{\mathcal{O}}_{i}(n+1, m+1, m+1)-\mathcal{V}_{i}-\mathcal{V}_{i}^{\prime} \\
\succ & -\mathcal{V}_{i}^{\prime} \widehat{\mathcal{O}}_{i}^{-1}(n+1, m+1, m+1) \mathcal{V}_{i},
\end{aligned}
$$

we can obtain

$$
\left[\begin{array}{cc}
\widehat{\Upsilon}_{i}(n+1, m+1, m+1) & 0 \\
* & \bar{\Upsilon}_{i}(n+1, m+1) \\
* & * \\
& \mathcal{V}_{i}^{\prime} \mathcal{A}_{i} \mathcal{L}_{i}(m+1) \\
& V_{i}^{\prime} A_{i} \\
& -\overline{\mathcal{O}}_{i}(n+1, m)
\end{array}\right] \prec 0
$$

with

$$
\begin{gathered}
\widehat{\Upsilon}_{i}(n+1, m+1, m+1) \triangleq \widehat{\mathcal{O}}_{i}(n+1, m+1, m+1)-\mathcal{V}_{i}-\mathcal{V}_{i}^{\prime} \\
\bar{\Upsilon}_{i}(n+1, m+1) \triangleq \overline{\mathcal{O}}_{i}(n+1, m+1)-V_{i}-V_{i}^{\prime}
\end{gathered}
$$

Then, by applying the congruence transformation to (44) by $\operatorname{diag}\left\{\mathcal{V}_{i}^{-1}, V_{i}^{-1}, V_{i}^{-1}\right\}$, and setting $Z_{i} \triangleq V_{i}^{-1}$, $H_{i}(n, m, m) \triangleq\left(V_{i}^{\prime}\right)^{-1} O_{i}(n, m, m) V_{i}^{-1}$, and $\overline{\mathcal{H}}_{i}(n, m) \triangleq$ $\left(V_{i}^{\prime}\right)^{-1} \overline{\mathcal{O}}_{i}(n, m) V_{i}^{-1}$, it can be obtained that

$$
\left[\begin{array}{cc}
\Phi_{i}(n+1, m+1, m+1) & 0 \\
* & \bar{\Phi}_{i}(n+1, m+1) \\
* & * \\
& \overline{\mathcal{A}}_{i} \mathcal{L}_{i}(m+1) \\
& A_{i} Z_{i} \\
& -\overline{\mathcal{H}}_{i}(n+1, m)
\end{array}\right] \prec 0
$$

where $\Phi_{i}(n+1, m+1, m+1)$ and $\bar{\Phi}_{i}(n+1, m+1)$ are defined in Theorem $1, \overline{\mathcal{A}}_{i} \triangleq \operatorname{diag}_{(M-1)}\left\{A_{i} Z_{i}\right\}$. Therefore, (45) can be ensured by (13) via replacing $A_{i} Z_{i}$ in (45) with $A_{i} Z_{i}+B_{i} U_{i}(\alpha)$. On the other hand, one can further derive (14) from (42) by pro- and post-multiplying $\left(V_{i}^{\prime}\right)^{-1}$ and $V_{i}^{-1}$. The proof is completed.

\section{E. Proof of Theorem 2}

Proof. (i) Because $\widetilde{\eta}_{i i}(\beta)=0$, (7) can be represented as

$$
\begin{array}{r}
\sum_{\beta \in \mathbb{N}_{+}} \rho_{i}(\beta) \sum_{j \neq i, j \in \mathcal{I}} \eta_{i j}(\beta)\left(A_{i}^{\prime}\right)^{\beta} P_{j}(n+1, \beta) A_{i}^{\beta} \\
-P_{i}(n, \alpha) \triangleq-Q_{i}(n, \alpha) \prec 0
\end{array}
$$

where $\eta_{i j}(\beta)$ and $\rho_{i}(\beta)$ are defined in (15). Then, it is straightforward to show that (46) is equivalent to

$$
\begin{aligned}
& \sum_{\beta \in \mathbb{N}_{+}} \rho_{i}(\beta)\left\{\sum_{j \neq i, j \in \mathcal{I}} \eta_{i j}(\beta)\left(A_{i}^{\prime}\right)^{\beta} P_{j}(n+1, \beta) A_{i}^{\beta}\right. \\
& \left.-\frac{1}{\widetilde{\rho}_{i}(\beta)} P_{i}(n, \alpha)\right\}=-\sum_{\beta \in \mathbb{N}_{+}} \frac{\rho_{i}(\beta)}{\widetilde{\rho}_{i}(\beta)} Q_{i}(n, \alpha) \prec 0 .
\end{aligned}
$$

Since $\widetilde{\rho}_{i}(\beta) \triangleq \sum_{\beta \in \mathbb{N}_{+}} \rho_{i}(\beta)=\sum_{\beta \in \mathbb{N}_{+}} \sum_{j \neq i \in \mathcal{I}} \pi_{i j}(\beta)=1$ and $\rho_{i}(\beta) \geq 0$, it can be observed that (46) can be ensured by (15).

(ii) From (16), it can be inferred that

$$
\begin{array}{r}
\sum_{m=0}^{\beta-1}\left(A_{i}^{\prime}\right)^{m}\left[\sum _ { j \neq i , j \in \mathcal { I } } \eta _ { i j } ( \beta ) \left\{A_{i}^{\prime} O_{j}(n+1, \beta, m+1) A_{i}\right.\right. \\
\left.\left.-O_{j}(n+1, \beta, m)\right\}\right] A_{i}^{m} \prec 0
\end{array}
$$

which can be rewritten as

$$
\begin{array}{r}
\sum_{j \neq i, j \in \mathcal{I}} \eta_{i j}(\beta)\left\{\left(A_{i}^{\prime}\right)^{\beta} O_{j}(n+1, \beta, \beta) A_{i}^{\beta}\right. \\
\left.-O_{j}(n+1, \beta, 0)\right\} \prec 0 .
\end{array}
$$

Combined with (17) and (47), the above inequality becomes

$$
\sum_{j \neq i, j \in \mathcal{I}} \eta_{i j}(\beta)\left(A_{i}^{\prime}\right)^{\beta} O_{j}(n+1, \beta, \beta) A_{i}^{\beta}-O_{i}(n, \alpha, \alpha) \prec 0 .
$$

Setting $P_{i}(n, \alpha)=O_{i}(n, \alpha, \alpha)$, one knows that (48) is equivalent to (15). This completes the proof.

\section{F. Proof of Theorem 3}

Proof. By Schur complement, (16)-(17) can be rewritten as

$$
\begin{array}{r}
{\left[\begin{array}{cc}
-\widetilde{\mathcal{O}}_{i}(n+1, \beta, m+1) & \widetilde{\mathcal{O}}_{i}(n+1, \beta, m+1) \mathcal{A}_{i} \widetilde{\mathcal{L}}_{i}(\beta) \\
* & -\widetilde{\mathcal{L}}_{i}^{\prime}(\beta) \widetilde{\mathcal{O}}_{i}(n+1, \beta, m) \widetilde{\mathcal{L}}_{i}(\beta)
\end{array}\right]_{\prec 0}^{(49)}} \\
{\left[\begin{array}{cc}
-\widetilde{\mathcal{O}}_{i}(n+1, \beta, 0) & \widetilde{\mathcal{L}}_{i}(\beta) \\
* & -O_{i}(n, \alpha, \alpha)
\end{array}\right] \prec 0}
\end{array}
$$

where $\widetilde{\mathcal{O}}_{i}(n+1, \beta, m) \triangleq \operatorname{diag}\left\{O_{1}(n+1, \beta, m), \ldots, O_{i-1}(n+\right.$ $\left.1, \beta, m), O_{i+1}(n+1, \beta, m), \ldots, O_{M}(n+1, \beta, m)\right\}, \mathcal{A}_{i}$ is defined in (43). Performing the congruence transformation $\operatorname{diag}\left\{\widetilde{\mathcal{O}}_{i}^{-1}(n+1, \beta, m+1) \widetilde{\mathcal{V}}, \mathbf{I}\right\}$ to (49) and $\operatorname{diag}\left\{\widetilde{\mathcal{O}}_{i}^{-1}(n+\right.$ $1, \beta, 0) \tilde{\mathcal{V}}, \mathbf{I}\}$ to $(50),(49)-(50)$ can be ensured by the following inequalities, respectively,

$\left[\begin{array}{cc}\widetilde{\Upsilon}_{i}(n+1, \beta, m+1) & \widetilde{\mathcal{V}}^{\prime} \mathcal{A}_{i} \widetilde{\mathcal{L}}_{i}(\beta) \\ * & -\widetilde{\mathcal{L}}_{i}^{\prime}(\beta) \widetilde{\mathcal{O}}_{i}(n+1, \beta, m) \widetilde{\mathcal{L}}_{i}(\beta)\end{array}\right] \prec 0(51)$ 


$$
\left[\begin{array}{cc}
\widetilde{\Upsilon}_{i}(n+1, \beta, 0) & \widetilde{\mathcal{V}}^{\prime} \widetilde{\mathcal{L}}_{i}(\beta) \\
* & -O_{i}(n, \alpha, \alpha)
\end{array}\right] \prec 0(52)
$$

with

$$
\widetilde{\Upsilon}_{i}(n+1, \beta, m+1) \triangleq \widetilde{\mathcal{O}}_{i}(n+1, \beta, m+1)-\widetilde{\mathcal{V}}-\widetilde{\mathcal{V}}^{\prime}
$$

where $\widetilde{\mathcal{V}} \triangleq \operatorname{diag}_{(M-1)}\{V\}, \mathcal{A}_{i}$ is defined in (43). Then, taking another congruence transformation $\operatorname{diag}\left\{\widetilde{\mathcal{V}}^{-1}, V^{-1}\right\}$ to (51) and (52), one will have

$$
\begin{array}{cc}
{\left[\begin{array}{cc}
\widetilde{\Phi}_{i}(n+1, \beta, m+1) & \widetilde{\mathcal{A}}_{i} \widetilde{\mathcal{L}}_{i}(\beta) \\
* & -\widetilde{\mathcal{L}}_{i}^{\prime}(\beta) \widetilde{\mathcal{H}}_{i}(n+1, \beta, m) \widetilde{\mathcal{L}}_{i}(\beta)
\end{array}\right] \prec 0(53)} \\
\\
{\left[\begin{array}{cc}
\widetilde{\Phi}_{i}(n+1, \beta, 0) & \widetilde{\mathcal{Z}} \widetilde{\mathcal{L}}_{i}(\beta) \\
* & -H_{i}(n, \alpha, \alpha)
\end{array}\right] \prec 0(54)}
\end{array}
$$

where $\widetilde{\Phi}_{i}(n+1, \beta, m+1)$ and $\widetilde{\Phi}_{i}(n+1, \beta, 0)$ are defined in Theorem 3, $H_{i}(n, \alpha, m)=\left(V^{\prime}\right)^{-1} O_{i}(n, \alpha, m) V^{-1}, Z=$ $V^{-1}, \widetilde{\mathcal{H}}_{i}(n+1, \beta, m) \triangleq \operatorname{diag}\left\{H_{1}(n+1, \beta, m), \ldots, H_{i-1}(n+\right.$ $\left.1, \beta, m), H_{i+1}(n+1, \beta, m), \ldots, H_{M}(n+1, \beta, m)\right\}, \widetilde{\mathcal{Z}} \triangleq$ $\operatorname{diag}_{(M-1)}\{Z\}, \quad \widetilde{\mathcal{A}}_{i} \triangleq \operatorname{diag}_{(M-1)}\left\{A_{i} Z\right\}$ and $\widetilde{\mathcal{L}}_{i}(\beta) \triangleq$ $\left[\sqrt{\eta_{i 1}(\beta)} \mathbf{I}, \ldots, \sqrt{\eta_{i(i-1)}(\beta)} \mathbf{I}, \sqrt{\eta_{i(i+1)}(\beta)} \mathbf{I}, \ldots, \sqrt{\eta_{i M}(\beta)} \mathbf{I}\right]^{\prime}$.

Note that (54) is the same as (19), and (53) can be ensured by (18) while replacing $\widetilde{\mathcal{A}}_{i}$ with $\widehat{\mathcal{A}}_{i}(\alpha)$.

\section{REFERENCES}

[1] H. Torikai and T. Saito, "Synchronization of chaos and its itinerancy from a network by occasional linear connection," Circuits and Systems I: Fundamental Theory and Applications, IEEE Transactions on, vol. 45, no. 4, pp. 464-472, 1998.

[2] Y. Ma, H. Kawakami, and C. K. Tse, "Bifurcation analysis of switched dynamical systems with periodically moving borders," Circuits and Systems I: Regular Papers, IEEE Transactions on, vol. 51, no. 6, pp. 1184-1193, 2004.

[3] Y. Takahashi, H. Nakano, and T. Saito, "A simple hyperchaos generator based on impulsive switching," Circuits and Systems II: Express Briefs, IEEE Transactions on, vol. 51, no. 9, pp. 468-472, 2004.

[4] A. P. Bowling, "Dynamic performance, mobility, and agility of multilegged robots," Journal of dynamic systems, measurement, and control, vol. 128 , no. 4 , pp. 765-777, 2006.

[5] J. T. Huang, T. V. Hung, and M. L. Tseng, "Smooth switching robust adaptive control for omnidirectional mobile robots," 2015.

[6] R. Yoshida, S. Imoto, and T. Higuchi, "Estimating time-dependent gene networks from time series microarray data by dynamic linear models with Markov switching," in Computational Systems Bioinformatics Conference, 2005. IEEE, 2005, pp. 289-298.

[7] Á. Halász, M. S. Sakar, H. Rubin, V. Kumar, G. J. Pappas et al., "Stochastic modeling and control of biological systems: the lactose regulation system of Escherichia coli," Automatic Control, IEEE Transactions on, vol. 53, no. Special Issue, pp. 51-65, 2008.

[8] L. Zhang, H. Gao, and O. Kaynak, "Network-induced constraints in networked control systems-A survey," Industrial Informatics, IEEE Transactions on, vol. 9, no. 1, pp. 403-416, 2013.

[9] P. Seiler and R. Sengupta, "An $H_{\infty}$ approach to networked control," Automatic Control, IEEE Transactions on, vol. 50, no. 3, pp. 356-364, 2005.

[10] L. Zhang, Y. Shi, T. Chen, and B. Huang, "A new method for stabilization of networked control systems with random delays," Automatic Control, IEEE Transactions on, vol. 50, no. 8, pp. 1177-1181, 2005.

[11] E. K. Boukas, Stochastic Switching Systems: Analysis and Design. Springer, 2007.

[12] O. L. V. Costa, M. D. Fragoso, and R. P. Marques, Discrete-time Markov jump linear systems. Springer Science and Business Media, 2005.

[13] R. Z. Khas'minskii, "Necessary and sufficient conditions for the asymptotic stability of linear stochastic systems," Theory of Probability \& Its Applications, vol. 12, no. 1, pp. 144-147, 1967.

[14] O. L. V. Costa and E. K. Boukas, "Necessary and sufficient condition for robust stability and stabilizability of continuous-time linear systems with Markovian jumps," Journal of Optimization Theory and Applications, vol. 99, no. 2, pp. 359-379, 1998.
[15] L. Zhang and J. Lam, "Necessary and sufficient conditions for analysis and synthesis of Markov jump linear systems with incomplete transition descriptions," Automatic Control, IEEE Transactions on, vol. 55, no. 7, pp. 1695-1701, 2010.

[16] P. Seiler and R. Sengupta, "A bounded real lemma for jump systems," Automatic Control, IEEE Transactions on, vol. 48, no. 9, pp. 1651-1654, 2003.

[17] L. Li and V. A. Ugrinovskii, "On necessary and sufficient conditions for $H_{\infty}$ output feedback control of Markov jump linear systems," Automatic Control, IEEE Transactions on, vol. 52, no. 7, pp. 1287-1292, 2007.

[18] C. Schwartz, Control of Semi-Markov Jump Linear Systems with Application to the Bunch-train Cavity Interaction. Ph.D. Thesis, Northwestern University, 2003.

[19] Z. Hou, J. Luo, P. Shi, and S. K. Nguang, "Stochastic stability of Ito differential equations with semi-Markovian jump parameters," Automatic Control, IEEE Transactions on, vol. 51, no. 8, pp. 1383-1387, 2006.

[20] J. Huang and Y. Shi, "Stochastic stability of semi-Markov jump linear systems: An LMI approach," in 2011 50th IEEE Conference on Decision and Control and European Control Conference (CDC-ECC), Dec 2011, pp. 4668-4673.

[21] L. Zhang, Y. Leng, and P. Colaneri, "Stability and stabilization of discrete-time semi-Markov jump linear systems via semiMarkov kernel approach," Automatic Control, IEEE Transactions on, DOI:10.1109/TAC.2015.2438424, 2015.

[22] V. Barbu and N. Limnios, "Empirical estimation for discrete-time semiMarkov processes with applications in reliability," Journal of Nonparametric Statistics, vol. 18, no. 7-8, pp. 483-498, 2006.

[23] R. A. Howard, "System analysis of semi-Markov processes," Military Electronics, IEEE Transactions on, vol. 8, no. 2, pp. 114-124, 1964.

[24] E. B. Dynkin, Theory of Markov processes. Courier Corporation, 2012.

[25] N. Limnios and G. Oprisan, Semi-Markov processes and reliability. Springer Science \& Business Media, 2012.

[26] J. Huang and Y. Shi, "Stochastic stability and robust stabilization of semi-Markov jump linear systems," International Journal of Robust and Nonlinear Control, vol. 23, no. 18, pp. 2028-2043, 2013.

[27] J. H. Vandermeer and D. E. Goldberg, Population Ecology: First Principles. Princeton University Press, 2003.

[28] P. Turchin, "Does population ecology have general laws?" OIKOS, vol. 94, no. 1, pp. 17-26, 2001. 